\title{
ZUR MITTELENGLISCHEN WORTBETONUNG.
}

In der nachfolgenden untersuchung beabsichtige ich die von Schipper in seiner englischen Metrik ${ }^{1}$ gegebene darstellung der mittelenglischen wortbetonung einer eingehenden prüfung $\mathrm{zu}$ unterziehen und im anschluss hieran seine auffassung der germanischen und mittelengl. langzeile zu beleuchten. Schipper's buch erschien zu derselben zeit, als meine ausgabe des King Horn fertig geworden war. Da ich mich in derselben bezuiglich der wortbetonung und der alten langzeile auf den standpunkt Lachmann's stelle, Schipper aber bezüglich beider punkte eine entgegengesetzte ansicht verficht, so musste es meine nächste aufgabe sein, Schipper's beweisfuhrung in allen punkten nachzuprüfen, um gewisheit zu erlangen, ob ich tatsächlich meine ausgabe, was das metrische anlangt, auf einen falschen boden gestellt hatte oder nicht. Ich glaube bei dieser nachprüfung mit vollkommener unbefangenheit verfahren zu sein und hoffe im folgenden nicht bloss eine vollständige widerlegung Schipper's, sondern auch einen nutzbaren beitrag zur mittelengl. metrik geliefert zu haben. Im anschluss an die theoretischen erörterungen werde ich mir alsdann erlauben, die auffassung, die Sch. der wort- und versbetonung des King Horn gibt, zu widerlegen und meine ausgabe im einzelnen ilım gegenüber zu rechtfertigen.

Schipper gründet seine mittelengl. wortbetonung nicht, wie man wol erwarten sollte, auf solche werke, welche das alte, nationale metrum, die germanische langzeile, in ihrer organischen fortentwickelung aufweisen, also auf werke wie Lazamon und King Horn, sondern auf nachbildungen fremder metren, metren, die nicht nach germanischer weise blos nach den heb-

1 Dr. J. Schipper, Englische Metrik in historischer und systematischer Entwickelung dargestellt. I. teil: Altenglische Metrik. Bonn, F. Strauss, 1581 . 
ungen gemessen werden, sondern auf dem prinzip der taktgleichheit beruhen, und deren nachbildungen im Englischen mit mehr oder weniger erfolg einen regelmässigen wechsel von hebung und senkung erstreben. Das bedeutendste dieser werke ist das Ormulum.

Es war ja äusserst verlockend, Orm's umfangreiches und metrisch genaues werk zum ausgangspunkt einer darstellung der mittelengl. wortbetonung aberhaupt zu machen. Aber die bedenken, die sich gegen ein solches verfahren erhoben, lagen doch auf der hand. Orm ist der strengste anhänger des ungermanischen prinzips eines regelmässigen wechsels von gehobenen und ungehobenen silben. Er kennt keine zweisilbige senkung, selbst keine schwereren fälle der verschleifung; es darf aber auch die senkung nirgends fehlen, es dürfen also nicht zwei betonte silben zusammentreffen: eine betonung wie gódspéll vermeidet er durchaus. Orm ist also eine erscheinung, die man, statt am anfange dieser entwickelungsperiode, erst am ende derselben hätte erwarten dürfen. In der tat vergeht geraume zeit, ehe das von ihm vertretene prinzip durchdringt, und die entwicklung der' englischen verskunst verläuft, von Orm abgesehen, ganz analog der deutschen in dem gleichen zeitraume, in welchem ja auch nur allmählich das prinzip des füllens der senkung, in der Lyrik zuerst, sich geltung verschafft, um dann freilich bis zur blossen silbenzählung auszuarten.

Auch wenn es also dem verf. gelungen wäre, alles, was er aus Orm beweisen möchte, tberzeugend darzutun, würde man doch unter hinweis auf die fremdartigkeit des von Orm befolgten versprinzips die allgemeingiltigkeit der aus dem 0 rmulum gezogenen schlüsse bestreiten können. Lassen wir indessen diesen gesichtspunkt vorläufig ausser acht und begntugen wir uns damit, auf diese bedenken hingewiesen zu haben.

Was Sch. aus Orm und gleichartigen gedichten dieser zeit vor allem beweisen möchte, ist die vollkommene gleichstellung aller tonlosen ${ }^{1}$ mit den stummen ableitungs- und flexions-

1 In meiner schrift: King Horn, Untersuchungen zur mittelengl. Sprach- und Literaturgeschichte, Strassb. 1876, unterschied ich s. 43 nur zwischen hochtonigen, tieftonigen und tonlosen silben, indem ich unter tonlosen silben alle diejenigen verstand, welche keine hebung zu tragen im stande sind, also alle silben mit (stummem, Lachmann) $e$ nach kurzer betonter silbe mit einfacher konsonanz: speke, cristene, und 
silben und damit die absolute hebungsunfähigkeit der ersteren.

Dieser punkt erschien ihm für die ganze englische wortbetonung und metrik so wichtig, dass er es für nötig erachtete, schon in der einleitung seines buches $\mathrm{s} .16 \mathrm{f}$. ausdrücklich hervorzuheben, dass 'an eine dem alt- oder mittelhochdeutschen gebrauche analoge verwendung der tonlosen flexionssilben im Englischen jedenfalls nicht zu denken sei'. Der beweis für diese behauptung wird im kap. 6 angetreten. Sehen wir, was Sch. $z u$ ihrer begründung vorbringt.

Zunächst meint der verf. s. 126, dass es von Orm, der bezüglich der quantität der vokale eine so genaue bezeichnungsmethode durchgeführt habe, zu erwarten gewesen, er würde, wenn wirklich ein so wichtiger unterschied in dem tone der endsilben zweisilbiger wörter vorhanden gewesen, diesen unterschied ebenfalls durch besondere zeichen angedeutet oder wenigstens durch verschiedene verwendung im rhythmus berücksichtigt haben. Was die verwendung im rhythmus betrifft, so wird hiervon gleich weiter die rede sein, und ich hoffe in der tat eine verschiedenartige behandlung tonloser und stummer endsilben auch bei Orm zu erweisen. Was aber das erste betrifft, so ist zu bemerken, dass der unterschied zwischen tonlosen und stummen silben im Mhd. und Me. nur durch die länge oder kürze der voraufgehenden silbe bedingt wird. Ausserdem möchte es schwer fallen, uberhaupt (obwol Orm seine verse 'nicht etwa nach der quantität einrichtete', s. Scb. a. a. o.) zu erweisen, dass Orm irgendwo die ton verschiedenheit zweier silben durch verschiedene zeichen ausgedruickt habe.

Betrachten wir die verwendung der silben mit unbetontem $e$ im versinnern. Zur füllung der senkung werden tonlose und stumme silben gleichmässig verwendet, bei Orm nicht anders, als im deutschen metrum, im Me. sowol als im Mhd. Man vergleiche z. b. Orm:

\section{King Horn:}

batt Godess Sune shollde wel;

unter tieftonigen silben alle mit dem nebenton behafteten silben, die im verse eine hebung tragen dürfen, zusammenfasste. Silben der letzteren art mit unbetontem $e$ werden also fiirder als tonlos, silben mit stummem $e$ als stumm, beide arten zusammen als unbetont bezeichnet werden. 
What he speke mizte, And told $e$ him ful zare;

Nibelungenlied :

Swie vil man gote diende.

Durchaus im irrtum befindet sich Sch., wenn er s. 139 aunimmt, dass nach der theorie von Jessen und andern nur "tonlose (sollte heissen "stumme") silben (kurzvokalische bei einfacher endkonsonanz) - wie etwa hafe ic 129, nimঠ aus nimed etc.' von der elision dürften betroffen werden, und nun daraus, dass Orm stumme und tonlose silben in dieser hinsicht gleich behandle, den schluss zieht, Orm kenne ubberhaupt keinen unterschied unter den silben mit unbetontem $e$. Orm legt sich in der behandlung der senkung gewisse beschränkungen auf. $\mathrm{Er}$ vermeidet die verschleifung auf der senkung (lied von K. H., einl. XIV, 3, b) und kennt keine verschleifung auf der hebung (a. a. o. einl. XVI, 3, b), verfährt aber sonst ganz den auch für deutsche metren feststehenden gesetzen gemäss, nach denen tonlose und stumme $e$ mit folgendem vokal (oder $h+$ vokal) verschmolzen werden (hífe off him, láefe off him; lüfẹ he tóc, lcée he tóc; shollde icc, shollde hé; vgl. lied von K. H., einl. XIV, 3, a und e, und XVI, 3, a). Auch die synkope, apokope und krasis, die Orm verwendet um einsilbige senkung, zu erlangen, widersprechen in nichts den germanischen metrischen prinzipien.

Was aber die hebungsfähigkeit tonloser endsilben anlangt, so verhält es sich damit folgendermassen. Betonungen zweisilbiger wörter wie wéndèn, lévè etc., die wir für das Me. in german. metren annehmen, werden iberhaupt im versinnern selten sein, da auf gehobenes tonloses $e$ widerum eine schwache silbe (im Mhd. nur mit unbetontem $e$ ) folgen darf, wie im K. H. etiva in den zeilen: hi rúngè pe béllè, in Hórnès ilikè, vielleicht auch fram kingè to kingè (vgl. s. 471 oben, und s. 477). Fur. Orm aber, der einen regelmässigen wechsel zwischen hebungen und senkungen beobachtet, sind sie geradezu unmöglich. Wol aber erlaubt sich Orm in solchen fällen, also bei einer durch position oder von natur langen, ersten silbe, eine tonversetzung ${ }^{1}$, wie dies beim auftakt auch im deutschen metrum gestattet war (s. lied von K. H. s. XVII), z. b.:

' Ich bezweifle, ob wir in allen diesen fällen, wie Sch. will, schwebende betonung annehmen dürfen. 
afflirr, Cristiss 10387, weddedd, vepést, oppnénn, sprungénn, sczzdé, waeré, twinné, seffné etc.

(vgl. auch Sch. s. 127). Von wörtern mit kurzer stammsilbe sind mir bei flüchtiger durchsicht des gedichtes in dieser verwendung nur aufgestossen: oférr 1034. 1693 und fadérr in der füf mal widerkehrenden zeile:

fadérr and sune and haliz gast

6772. 10990. 11178. 11517. 18645. Man kann hier an verlängerung des vokales oder einfluss des schwereren schlusskonsonanten denken, bei oferr ist auch die analogie von underr, dedikation $9 \mathrm{u}$. oft, nicht ausgeschlossen, bei faderr das formelhafte der zeile nicht ausser acht zu lassen. Schwerlich aber werden sich tonversetzungen wie zifé, bodénn, borénn, godé etc. nachweisen lassen; im auftakt wenigstens begegnen sie nicht.

In dreisilbigen wörtern bei betonter erster, langer silbe war die regelmässige tonfolge típënndè, wintrèden in Orm aus bereits bekannten gründen nicht möglich; hier musste eine tonversetzung eintreten. Wenn nun, wie Sch. will, tonlose und stumme silben gleichwertig waren, so wäre für Orm die einfachste und fast allein zulässige tonversetzung die von der zweiten auf die dritte silbe gewesen, so dass erste und dritte silbe den ton erhielten. Dies ist aber nicht der fall. Mit ganz verschwindenden ausnahmen trifft der ton die zweite silbe, entsprechend german. regel (vgl. lied von K. H. XVIII, 2). Die beispiele sind zahlreich:

Cristéne, hapène (daneben hćpenn, doch kein hêpene), brasine, firéne, gildéne; sexténe, pritténe; loeréde (neben lóredel), fullhtnéde, fullhtnédenn, tacnède, trowwéde, shifftédenn, strenéde, wundrédenn; boernénnde, fifténnde, pritténnde, pusénnde, pehténnde, hoelénnde; Englisshe, Judisskenn, mennisske etc.

Nur in cniddedénn 8613 und filledénn 14040 hat die schwerere endung die eigentlich tonberechtigte silbe tuberwogen; in góddspellés ist auch die dritte silbe nach unserer theorie tonfähig.

Bei betonter, erster kurzer silbe aber ist die german. betonung, die ja in diesem falle auch fur Orm passte, geblieben:

lúfedé 16712. 17033, clépedénn 12978, pó!edé 11822, widevé 8632, nizhenndé 4488, séfenndé 4168. 4464 neben séoffnde,

meistens mit folgender schwacher silbe in der senkung, so dass man annehmen darf, dass für Orm auch dieses gesetz german. metrik (s. o.) noch fühlbar gewesen ist. Es begegnet 
keine tonversetzung auf die zweite silbe, wie es bei langer stammsilbe die regel ist.

In wörtern mit tieftoniger silbe an der dritten stelle ist die regelmässige betonung fast durchgängig gewahrt. Tonversetzungen wie afftérrward, fowwértiz sind erklärlich. Es findet sich jedoch kein bodéword, seofénntiz u. s. w.

In allen diesen fällen, von den vier besprochenen ausnahmen abgesehen, trifft also die tonversetzung ein tonloses, nach unserer theorie hebungsfähiges $e$. Und schon hiermit wäre ein unterschied in der behandlung des tonlosen und des stummen $e$ auch bei Orm nachgewiesen.

Weiter aber beobachtet Orm einen unterschied zwischen tonlosen und stummen endsilben darin, dass er am schlusse des septenars, wie schon Jessen hervorgehoben und mir eine erncute prüfung des gedichtes bestätigt hat, ausschliesslich nur zwei- oder mehrsilbige wörter mit tonloser ${ }^{1}$ endsilbe verwendet, also zweisilbige wörter, deren erste, oder dreisilbige, deren erste und zweite silbe von natur oder durch position lang ist. Ausnahmen von dieser regel gibt es nicht, obwol Schipper deren s. $129 \mathrm{f}$. anführt, die ich sofort bespreche.

lŏfe, das Jessen (Grundzüge der altgermanischen Metrik, Zeitschr. f. deutsche Philol. von Höpfner und Zacher II, 138 ff.) als gegen seine beobachtung sprechend nambaft macht, und das Schipper, Jessen citierend, für sich in's feld führt, beruht auf einem irrtum. In der von Rev. Robert Holt besorgten zweiten ausgabe des Ormulum von R. M. White ${ }^{2}$ steht z. 1445 das richtige lafe, mit langem stammvokal. Ausserdem führt nun Sch. a. a. o. noch weitere versausgänge mit angeblich kurzer stammsilbe auf, darunter merkwürdiger weise defell, deofell = ac. deófol; metedd, p. p. von ae. mîctan pingere, s. 211 bei Ettmüller, und wake, pl. von ae. wàc, ahd. weih. Es bleiben dann

1 Also mit unbetontem $e$ nach langer silbe. Mit tönendem vokal begegnet nur das adv. wukemalumm 534 und 536. Sonst vermeiden Orm sowol wie die iibrigen me. dichter, die sich des gleichen metrums bedienten, tieftonige silben, jedenfalls aus dem grunde, weil sonst ihre leser der schlusssilbe eine volle hebung gegeben hätten.

${ }^{2} \mathrm{Da}$ mir die erste ausgabe von White nicht zu gebote steht, vermag ich nicht zu entscheiden, ob sich Jessen blos verlesen, oder ob White in der tat an der angegebenen stelle lŏfe hat, das dann Holt nach der hs. corrigiert hätte. (White hat lofe. R. W.) 
noch zede, das vielleicht schon ae. als ze-eóde anzusetzen ist; litell, das walurscheinlich unter nordischem einflusse verlängert ist, wie die schreibung litell andeutet, und size, bei welchem worte die schreibung size 11421. 11481 gleichfalls auf verlängerung, die schreibung sizze (in sizzefast) auf aussprache des $z$ als doppelkonsonanz hindeutet, wie in twizzes, bezzen, fazzerr, ezze, die alle als versschliusse vorkommen. ${ }^{1}$ Von dreisilbigen wörtern begegnen mit versetzter betonung wol hoelénnde, dagegen kein hrepène, gildéne, oppriédenn etc., da hier die letzte silbe stumm wäre.

Wenn also in einem werke von mehr als zehntausend zeilen nur klingende versschlüsse sich finden, so kann das nicht auf einem zufall beruhen. Schipper will dieses misverhältniss, das zwischen den klingenden und stummen ausgängen (wie ich gezeigt habe, gibt es stumme ausgänge tiberhaupt nicht) herrscht, s. 128 damit erklären, dass 'die summe der langvokalischen ebenso wie die summe der durch position langen wortstämme zusammengenommen die summe der wortstämme mit kurzem stammrokal bei einfacher auslautender konsonanz' erheblich ubertreffe. Diese begründung würde, selbst wenn alle von ihm aufgeführten versschllisse wirklich kurze stammsilben hätten, durchaus nicht genligen. Worte wie spekenn, zifenn,

' Von offe sucht Sch. s. 131 wahrscheinlich zu machen, dass Orm hier dem metrum zu liebe ein $e$ an das gewöhnliche off gebängt habe. offe begegnet jedoch auch im versinnern, ebenso wie onne, und zwar beide in adverbialer geltung (als postpositionen), wie stets im versausgang. offe kommt ausserdem auch sonst vor, z. b. in der hs. $O$ des K. H., s. glossar s. 134 und bei Stratmann. Aus dieser form jedoch schliessen zu wollen, dass Orm die verdoppelung des konsonanten, anch wo sie ursprünglich ist, als solche nich t empfunden, sondern darin nur ein zeichen der kürze des voraufgehenden konsonanten gesehen habe (Sch. s. $131 \mathrm{f}$.), ist mehr als gewagt. Ich glaube vielmehr umgekehrt, dass Orm die kürze des vokals in offener silbe nur darum nicht durch verdoppelung des konsonanten (in wörtern wie bere, nime, zifenn etc.) bezeichnet hat, weil sonst die gefahr, den konsonanten doppelt zu sprechen, unvermeidlich gewésen wäre, was dann auch auf die qualität des folgenden stnmmen $e$ eingewirkt hätte. Wenn nun der verfasser aus dieser falschen prämisse weiter den schluss zieht, dass für Orm auch in wörtern wie libben, seggen etc. die betonte silbe als kurz anzusehen und darum die endsilben als absolut tonlos (d. h. nach unserer bezeíchnung stumm) zu betrachten seien (s. 132), so bedarf dies keiner weiteren widerlegung. 
tredenn, farenn, talienn, sune, gode, bode, wune etc. waren so bequeme und naheliegende versschlüsse, dass sie der um passende schlussworte so oft verlegene dichter sicher nicht verschmäht hätte, wenn er sie nicht hätte vermeiden wollen. Die weitere erklärung Sch.'s auf s. 134 ist in ihrer begründung unzutreffend, denn Orm hat durchaus nur die uncontrahierten participalformen borenn, froren, forboren; praktisch deckt sie sich mit unserer ansicht von dem charakter stummer silben.

Nicht anders nun verhält sich das zweite der von Sch. zu seiner beweisführung herangezogenen gedichte, das Poema Morale, nur dass dieses sich noch enger an die altnationale verskunst anschliesst. Hier wird kein regelmässiger wechsel von hebungen und senkungen erstrebt. Zwischen zwei hebungen kann die senkung fehlen (s. auch Sch. s. 144). Tonversetzung findet darum fast nur am schlusse der ersten vershälfte oder der zeile statt (z. b. wallínde, endinge etc., vol. lied von K. H. einl. s. XVIII). Andrerseits ist zweisilbigkeit der senkung unter den in der einleitung zum lied von K. H. gegebenen grenzen gestattet. Die tonlosen $e$ sind im innern des verses noch durchaus in der alten weise als tonfähig behandelt, wenn auch die beispiele nicht gerade häufig sind. Man vgl. (meine zablen bezielıen sich auf den abdruck der Lamb.- bezw. Egerton-hs. in Old Engl. Hom. I. Ser. ed. Rich. Morris, s. 159 ff.) 291 hédènemén, 293 cristènemén, 351 mid énglène sónge, 267 and po pet wéren gétsères, nicht gítserès, wie Sch. s.' 143 betont; 319 zif we séruèden gód(e), 258 po pe sünegède $(n)$ múchel.

Zahlreiche zweisilbige ausgänge mit stummem $e$ am schlusse der ersten vershälfte beweisen, dass der dichter des P. M. noch in alter weise eine letzte stumme silbe der voraufgehenden hebung zurechnet, diese ausgänge also einsilbigen rhythmisch vollkommen gleich erachtet. ${ }^{1}$ Unter den 396 zeilen des gedichtes finden sich deren mehr als achtzig, ein weiterer beweis, dass ihr nichtvorkommen am schlusse der zeile bei Orm und, wie wir sehen werden, im P. M. nicht in dem zustande der sprache begründet sein kann. Dass Orm dieselben nicht verwendet, hat meines erachtens seinen grund allein in der von ihm angestrebten, ubermässigen korrektheit. Beispiele aus dem P. M. sind:

1 Vgl. hierzu auch Einenkel, Anglia, Anz. zu b. V, s. 33 f. 
dede 2, dude 96, iquede 9, stude 24. 43. \$6. 295, biforen 26. 158 , were 31 , forzelen 34 . 9S, binimen 44. 48. 50, kare 45, berej 46, drazen 49, luue 56. 58. 189. 317. 334, muchel 22. 60 . 62. 113. 211. 353. 365. 389, haucd 65. 70. 357, zcue (sbst.) 74, binopen 87 , fole ( $=$ feole) 97. 286. 356, wrile (sbst.) 100, biJeten 105, iwriten 118, dure 124. 127, later 131, wuned 136, speken 145 , cumen 154 etc.

Daneben hat das P. M. allerdings auch eine beträchtliche anzahl von ersten vershälften mit vier hebungen und klingendem ausgange. Dieselben erledigen sich jedoch fast alle dadurch, dass das iberfliessende $e$ auf folgenden vokal oder auf $h+$ vokal verschliffen wird, so in:

tilpe is 57, go de and 61, horde and 85, hèsẹ and 91; ferner in 147.

151. 156. 204. 212. 214. 228. 242. 253. 272. 276. 301. 305. 312. ${ }^{1}$

In einer geringen anzahl von fällen möchte man an verschleifung mit der folgenden senkung denken, so:

of pine pe 133, dome pet 155, deme pa 190, wise po (nach der Digby-hs.), pine ne 290.

In allen andern fällen gibt die heranziehung der ïbrigen hss. das richtige. Sch. hat, wie es scheint, seine beobachtungen auf den von Zupitza im 1. bande der Anglia veröffentlichten text der Digby-hs. A 4 beschränkt, sonst würde er gesehen haben, dass vershälften wie: And on pos lopes diefles werkes, per inne sended to bet loueden, he on is muchele more and betere (s. $140 \mathrm{f}$.) einer korrektur durch die übrigen hss. bedürfen. Es unterliegt gar keinem zweifel, dass eine kritische ausgabe des gedichtes einen metrisch vollkommen korrekten text liefern könnte. ${ }^{2}$

Während also die erste vershälfte stumpfen, und zwar, ganz nach der alten weise, ein- oder zweisilbig stumpfen, klingenden ausgang aber nur dann zeigt, wenn möglichkeit der verschleifung vorhanden, ist der schluss der zeile widerum wie im Ormulum nur klingend, d. b. zweisilbig mit erster, langer silbe und folgendem tonlosen $e$, oder dreisilbig mit kurzer betonter silbe an erster stelle, z. b. 25-26 houene : souene, $103 \mathrm{ff}$. forsworene : icorene : iborene : forlorene. Zwar weiss Sch. s. 135 auch hier einige versschlüsse als 'entschieden kurzvokalische'

1 Ich will nicht unerwähnt lassen, dass auch eine beträchtliche anzahl stummer $e$ auf diese weise verschliffen werden können.

${ }_{2}$ Die kritische ausgabe des P. M. von H. Lewin, welche mir zu spät zukam, scheint gerade auf diese verhältnisse nicht genügend rücksicht zu nehmen. 
und dennach mit folgendem stummen $e$ nambaft zu machen, doch widerum sehr mit unrecht; denn imeten, Lamb.-lıs. z. 131, 237, ist ae. gemêtan, convenire, Ettm. s. 198, das darauf reimende beten ist ae. bêtan emendare; stelen: helen aber z. 159, 160 sind plur. prät. ae. slêclon, hêclon. ${ }^{1}$

Es kann also gar keinem zweifel unterliegen, dass in diesen beiden. hervorragendsten me. nachbildungen des katalektischen jambischen tetrameters (septenars) mit absicht nur ausgänge mit tonlosem $e$ in der letzten silbe verwendet sind. Der grund hierzu ist nicht schwer zu erkennen. Zweifelsohne wurde in dem lat. vorbild die letzte silbe als zu dem vierten unvollständigen takte gehörend empfunden, der durch die notwendig eintretende pause sein volles maass erhielt. In den englischen nachbildungen musste gleichfalls die letzte silbe als einem weiteren takte zugehörend hervortreten. Dies war aber nur möglich wenn die voraufgehende betonte silbe lang war. Nach einer kurzen stammsilbe würde ein folgendes (stummes) $e$ zur voraufgehenden hebung gehörig betrachtet worden sein, so dass in diesem falle die letzte balbzeile nur drei hebungen stumpf erhalten hätte. Andrerseits konnten tieftonige silben darum nicht verwendet werden, weil dieselben am schlusse der zeile notwendig eine volle hebung tragen müssen. Worte wie wimman, ofsprung, ending etc. konnte das P. M. am schlusse der ersten hälfte mit zwei hebungen gebrauchen, doch nicht am ende der zeile, weil alsdann der katalektische charakter des metrums verloren gegangen wäre. Zwischen diesen beiden, den stummen und tieftonigen silben, stehen nun die silben mit tonlosem $e$ mitten inne, und gerade dieses eigentümliche verhalten der besprochenen me. gedichte liefert uns einen neuen und durchschlagenden beweis fur die allgemeingiltigkeit der von Lachmann für das Germanische festgestellten tonabstufungen.

Fassen wir das resultat unserer bisherigen untersuchung zuisammen, so lautet es:

1. Die senkung wird in beiden gedichten, von 0rm unter gewissen beschränkungen, nach den für das deutsche metrum nach unserer the orie guiltigen gesetzen behandelt.

2. Beide gedichte machen zwischen tonlosen und stummen silben einen unterschied in der art, dass

1 Vgl. auch Einenkel a. a. o. s. 51 anm. 
a) in Orm von notwendig werdender tonversetzung mit verschwindenden ausnahmen nur tonlose silben, nie ein schliessendes stummes $e$ betroffen werden, das P. $M$. aber ganz in der alten weise tonlose silben als heb. ungsfähig verwendet;

b) Orm und das P. M. nur silben mit tonlosem $e$ fül fähig erachten, die letzte, den katalektischen versschluss anzeigende senkung zu bilden.

Unser ergebniss bildet also in allem den direkten gegensatz zu Schipper's resultat, welches s. 141 darin gipfelt, dass die flexionssilben $z$ weisilbiger wörter, einerlei ob dieselben lange oder kurze stammsilben haben mögen, tonlos in seinem sinne (d. h. unfähig einen ton zu erhalten) seien. Auf welcher seite hier die wahrheit liege, wird nach unserer betrachtung wol niemanden mehr zweifelhaft sein.

Bis zu diesem punkte dürte also Schipper's versuch, die absolute tonunfähigkeit aller silben mit unbetontem $e$ zu erweisen, als vollständig misgltuckt anzusehen sein. Nun sucht aber Sch. seine resultate durch heranziehung einer weiteren reihe poetischer werke dieser epoche zu stiitzen, deren metrische eigentümlichkeiten auf den ersten blick geeignet scheinen, ihm recht zu geben. Es sind dies gedichte, welche die ersten nachbildungen romanischer metren auf englischem gebiete enthalten. Wir mussten schon bei beginn unserer betrachtung unsere bedenken dartiber äussern, dass Sch. zum ausgangspun]ste seiner metrischen untersuchungen, denen er allgemeine giltigkeit zu geben gedachte, nachbildungen eines latein. metrums wählte. Immerhin hatte man es bier mit einem vorbilde zu tun, dessen eigentumlichkeiten man, so gut es gieng, dem heimischen idiom anpassen konnte, ohne die ïberlieferten sprach- und rhythmischen gesetze direkt zu verletzen. Die im Englischen in dieser epoche nachgeahmten romanischen metren aber zeigten gerade in dem punkte, der bei unserer gegenwärtigen untersuchung besonders in betracht kommt, eine sofort in die augen und in's gehör fallende abweichung von den german. grundgesetzen der verskunst. Sie misachteten das gesetz, das auf die letzte betonte silbe des verses keine tonfähige silbe mehr folgen dürfe. In den hier zunächst in betracht kommenden metren, dem achtsilbigen, zum sogenannten kurzen reimpaare romanischer herkunft verwendeten verse und dem alexandriner wurde die überfliessende silbe am schlusse, im alexandriner 
auch vor der Cäsur, nicht gezählt, also männlicher und weiblicher ausgang gleich geachtet. Dies ahmte man in den englischen nachbildungen getreu nach. Man kann also im grunde genommen auch hier nicht sagen, die unbetonten silben wurden gleich behandelt, der unterschied zwischen tonlosen und stummen silben fiel fort (der freilich nach Sch. nie bestanden), das hiesse der sache eine durchaus falsche deutung geben; im versausgang wird eine letzte unbetonte silbe einfach nicht $\mathrm{mehr}$ gez ählt. In der tat begegnen in allen hierber gehörenden gedichten stumpfe, sei es ein- oder zweisilbige, und klingende ausgänge neben einander.

In dem englischen Paternoster (Old Engl. Hom. I. Ser., s. 55 ff.), welches in dem sog. kurzen (viertaktigen) reimpaare romanischer herkunft gedichtet ist, finden sich bei der gleichen anzahl gehoben ersilben versausgänge wie is : ivis, beren : weren (inf.), iborene : icorene, ibeden : reden, rihte : wihte; in der den alexandriner nachbildenden Passioun of our Lord (An Old Engl. Misc. ed. Rich. Morris) ausgänge wie bigon : ouercom, furlore : peruore, dede: lede, dryhte:myhte, vadere : togadere etc. nebeneinander.

Man ist um so weniger berechtigt hier von einer gleichstellung der tonlosen und stummen silben zu reden, als im innern des verses in diesen gedichten betonungen nach germanischer weise noch gestattet sind.

Man vgl. aus der Passioun peóuène dich 80, gýnène king 348, hélàre 115, chéffàre 116.

In dem gedichte Doomsday, in septenaren und alexandrinern (Old Engl. Misc. s. 162 ff.), begegnet eine erste vershälfte pát mákedé pe quéd, dieselbe zeile in dem gedichte Death (a. a. o. $168 \mathrm{ff}$.$) z. 187$. In dem letzteren gedichte begegnen ferner betonungen wie sittèð ischrüd 9, pénchèd iwis 13, hérèd iwis 21, vórmès ifére 82 (zweite halbzeile), siehe anhang no. 13, 14. Man beachte, dass auch hier überall auf die gehobene tonlose silbe eine schwache senkung folgt.

Also auch diese gedichte erweisen sich bei näherer betrachtung den behauptungen Schipper's nicht günstig. Man kann ja zugeben, dass die englische sprache auf dem wege war, den unterschied zwischen tonlosen und stummen silben auszugleichen, ja dass sie auch ohne fremden einfluss dazu gekommen wäre, alle unbetonten endsilben gleich zu behandeln. In dieser zeit aber wurde ihre metrische verschiedenheit noch deutlich empfunden und noch lange erhalten sich ganz beträchtliclie spuren davon in den nationalen poetischen formen. Schipper 
aber glaubt diesen unterschied nicht nur für die von ihm behandelten fremdmetrischen, sondern für alle me. poetischen werke, ja nicht bloss für diese epoche, sondern überhaupt für das ganze gebiet des Englischen leugnen zu dürfen.

Auf die bei seinem beweise herangezogenen metren beschränkt waren seine resultate von geringer praktischer bedeutung. Dass in den aus dem Romanischen entlehnten metren stumpfer und klingender versausgang gleiche berechtigung hatten, wurde ja bisher gar nicht bezweifelt. Auch für den tetrameter an sich war es von geringer bedeutung, ob der ausgang ein stummes oder ein tönendes $e$ zeigte. Bedeutung, höchste bedeutung erlangten die ergebnisse seiner untersuchung erst, wenn sie auf rein deutsche metren übertragen wurden. Und Sch. zögert keinen augenblick dies zu tun. Während er s. 125 noch verspricht, die ungiltigkeit der Lachmann'schen regeln auch für Lazamons Brut und den King: Horn nachzuweisen, begnügt er sich bei besprechung dieser gedichte, s. 157 und s. 182, auf den im 6. kapitel angeblich für das Altenglische geführten nachweis hinzudeuten und glaubt sich der mühe überhoben 'die auf grund der Lachmann'schen gesetze aufgebaute theorie 'Trautmann's über das wesen und die entstehung des Lazamon-verses im einzelnen zu widerlegen'. Von den in meinen untersuchungen zu King Horn aufgestellten prinzipien der mittelenglischen wortund versbetonung ist dann gleichfalls weiter keine rede mehr.

Ich habe bisher durchaus nur mit Sch.'s eigenem material operiert und mich in meiner widerlegung auf die von ihm berlicksichtigten werke beschränkt und diese nur etwas gründlicher ausgebeutet. Nur der vollständigkeit wegen will ich zum schlusse noch der behandlung des versausganges im King Horn gedenken, obwol ich den lesern dieser zeitschrift damit nichts neues sagen werde; doch möchte ich damit dartun, dass Sch. auf diese verhältnisse hätte eingehen müssen, wenn sein beweis wirksam ausfallen sollte.

Zunächst haben im K. H. alle zeilen mit einsilbigem, stumpfen schluss vier hebungen (Ańd his góde knizles twó $A^{\prime} l$ to féve wére $b \dot{o}$ ), desgleichen, von wenigen, später zu besprechenden ausnahmen abgesehen, alle verse mit schliessendem, stummen $e$ (z. $23 \mathrm{f}$. A'lle riche mánnes sünes And álle swipe faire gumes, vgl. ferner z. 77 f., 165 f., 201 f., 585 f., 605 f., 1471 f.), die letzte stumme silbe wird auf der hebung verschliffen. Macht 
dagegen ein dreisilbiges wort den schluss mit kurzer, betonter erster silbe, so hat die zeile nur drei hebungen, die vierte trifft das letzte, tonlose $e$ (z. $685 \mathrm{f}$. Crist and seint Stévenè, Türne pine swéuenè, vgl. 1043 f.). Bei zweisilbigem ausgang und betonter erster langer silbe haben wir gleichfalls drei hebungen, die vierte ruht auf der schliessenden tonlosen silbe (A'lle béon he blibè). Reime wie time: bi me $549 \mathrm{f}$., sitte: mitte (für mit be $643 \mathrm{f}$ ) mögen in dieser zeit immerhin noch für die tonfähigkeit der endsilben in's gewicht fallen (vgl. Sch. s. 185). Bei dreisilbigem ausgang mit betonter erster langer silbe und folgender kurzer silbe trägt diese letztere die vierte hebung und die letzte silbe ist stumm (1153 f. pu wénst ich béo a béggère Ańd ich ám a fissère); ist auch die zweite silbe lang, so fällt auf diese, wenn nicht tonversetzung eintritt, bereits die dritte bebung, die vierte ruht auf dem letzten, tonlosen $e$ (z. 69 Of álle wimmànnè, $177 \mathrm{He}$ wás pe fairèste, $179 \mathrm{We}$ beóp of Súddeïnè (vgl. King Horn, unters. s. 45). Das sind so unverkennbare, in die augen springende unterschiede, die dabei genau den german. betonungsgesetzen entsprechen, dass es schwer fallen muss, dieselben nicht zu bemerken.

Wenn wir nun fragen, warum Sch. die tonlosen silben zu absolut tonunfähigen machen und sie auf diese weise zu stummen silben herabdrücken möchte, so lautet die antwort: um im Me. ein hauptsächliches argument für seine theorie der german. langzeile zu gewinnen. Schipper ist unbedingter anhänger der von Vetter ${ }^{1}$ mit einigem glück geltend gemachten ansicht, dass der regelmässige alliterierende langvers aus zwei durch den stabreim verbundenen gliedern oder halbversen von je zwei hebungen bestehe. Zur unterstlitzung dieser ansicht hat Sch. auf dem gebiete des Ae. nichts wesentlich neues beigebracht. Er beschränkt sich darauf, in einer anm. zu s. 46 vier argumente aus Vetter's schrift für diese theorie zusammenzustellen, argumente, die sich nur negativ gegen die Lachmann'sche theorie wenden, aber positiv für die theorie der vier hebungen nichts erweisen.

Dagegen sucht Sch. vom Me. aus jener theorie neue stiitzen zuzuführen durch den von uns bereits als misglückt erkannten nachweis der absoluten tonunfähigkeit unbetonter silben.

${ }^{1}$ Ferdinand Vetter, Ueber die german. Allitterationspoesie. Wien 1872. 
War diese tonunfähigkeit erwiesen, so fiel damit das gesetz, dass im german. metrum bei klingendem ausgange die letzte hebung auf der klingenden silbe ruhe. Es fielen ferner zahlreiche (neben-)hebungen im innern des verses. Zahlreiche verse, wie feollen ba foeie, madmes inoze etc. aus Lazamon, die wir nach dem schema" ' 1 lesen, konnten nunmehr bloss mit zwei hebungen gelesen werden. Von da aus war es dann leicht wahrscheinlich zu machen, dass, um die gleichbeit des rhythmus zu bewahren, auch in versen mit drei oder vier hochtonigen worten nur zwei hebungen zu lesen seien, also breo scipen góde, umbe fiftene żer; áfre al swa heore lif. Denn für die senkung hat die zweihebungstheorie eine grenze nur nach einer seite: sie kann an jeder stelle fehlen, doch muss jede halbzeile mindestens eine senkung haben; nach der andern gibt es keine beschränkung, denn ein maximum von sieben silben kann man kaum als eine beschränkung ansehen. So will denn Sch. im Lazamon betonen:

On Itálize heo comen to lónde; pat maiden wes ihoten Láuine; Sáturnus heo ziuen sátterdoei etc.

Die senkung ist denn auch der eigentliche wunde punkt der zweihebungstheorie. Wenn Vetter gegen Lachmann's versbetonung geltend macht, dass bei ihier konsequenten durchfluhrung die senkungen schliesslich in vielen fällen ganz verschwinden, dass verse anzunehmen sind, die nur aus gehobenen silben bestehen, so misssen wir umgekehrt gegen seine theorie den vorwurf erheben, dass in ihr die senkung jeder gesetzmässigen ordnung entbehre. Es fragt sich, welcher vorwurf schwerer wiegt. Zunächst müssen auch die anhänger der gegnerischen theorie (so auch Sch. an vielen orten) zugestehen, dass im germanischen metrum die senkung zwischen zwei hebungen fehlen darf. Wenn dies an einer oder an zwei versstellen geschehen kann, warum nicht an allen? Ein rhythmus bleibt auch dann noch bestehen, da nie alle gehobenen worte die gleiche tonhöhe haben, sondern gewöhnlich zwei hauptund zwei nebenhebungen vorhanden sind. Also braucht uns

1 Es kommt auch hier bei den mit dem nebenton versehenen silben in erster linie auf die füllung des taktes an, die wir nicht so sehr durch den ton, als durch volle, getragene aussprache der nebentonigen silben erreichen, so dass wir das schema vielleicht besser wie folgt darstellen: , - - (z. b. in Hórnes ilikē). 
selbst das von Vetter angefihrte beispiel einer aus einem worte bestehenden halbzeile randwîggendra nicht zu schrecken (s. Sch., s. 47, anm. c). Wol aber geht nach meiner ansicht bei annahme der zweihebungstheorie jeder vernünftige rhythmus verloren. Man lese nach dieser die aus Beowulf beliebig herausgegriffenen zeilen:

veóx under vólcnum veórd-myndum päh; fýren-pearfe ongeát Beoinulf was bréme; leof leod-cyning; ne gefeah he pare fóhðe ete.

oder. gar aus andern dichtungen zeilen wie úp to ban ćlmihtigan gode, um sich zu uberzeugen, dass wir mit der annahme von Schipper's und Vetter's theorie die absolute formlosigkeit des germanischen metrums begründen.

Dass die altgermanische langzeile auf ein indogermanisches metrum zurückgeht, welches nur auf der quantität beruhen konnte, ist wol ziemlich allgemein zugestanden. Erst im Germanischen wurden gewisse versstellen durch den wortton noch besonders hervorgehoben und schliesslich unter diesen gehobenen silben einige wider durch den äusserlichen schmuck des stabreimes ausgezeichnet. So falsch es nun wäre, in den modernen, durch den endreim ausgezeichneten poetischen formen in diesem äusserlichen schmuck- oder bindemittel das ausschlaggebende moment der poetischen form zu erkennen und alles andere für nebensächlich anzusehen, ebenso falsch ist es meines erachtens, wenn man den stabreim zum ausschliesslichen kennzeichen einer poetischen form macht. Dies aber geschieht von seiten der vierhebungstheoretiker. Zwei gehobene silben in jeder balbzeile, das ist so ziemlich das einzige erforderniss, das sie an den rhythmus der verses stellen; fur die senkung wird weder ein maass, noch ein bestimmter rhythmus festgesetzt. Das eigentlich bedeutsame ist der reim. Nun vermochte der stabreim wol den rhythmus in bedeutendem maasse zu beeinflussen, indem er die reimenden worte vor den-ubrigen besonders hervorhob, ja, ich will zugeben, dass er im stande war, das ursprüngliche maass des verses zusammenzupressen, wie dies tatsächtich im Altnordischen geschehen ist, wo darum auch das altgerman. metrum zur epischen erzählung untauglich geworden ist; aber der rhythmus selbst gieng darum nicht verloren. Wie wäre es sonst möglich, dass auch verse ganz ohne den stabreim bestehen konnten, wie sie der ae. psalter auf- 
weist? Wie wäre es möglich, dass in späterer zeit der stabreim silben trifft, die nach der zweihebungstheorie in der senkung stehen, wie bei Aelfric? Dem entspricht, dass mit dem zerfall der alliteration und dem eindringen des endreimes der viertaktige charakter der halbzeile immer deutlicher hervortritt, den Sch. schliesslich selbst nicht mehr leugnen kann (s. 161 und besonders s. 162), wenn er diese erscheinung auch auf romanischen einfluss zurückführen möchte; ohne sie doch widerum direkt von einem romanischen vorbilde abzuleiten.

$\mathrm{Zu}$ welchen widersprüchen und absonderlichkeiten Sch. durch seine theorie gefuhrt wird, mag noch im folgenden gezeigt werden. Während er für Lazamon hier und da die möglichkeit einer skansion nach Lachmann'scher art zugibt, aber dennoch behauptet, dass jene verse, im zusammenhange des ganzen passus gelesen, notwendig denselben zweihebigen rhythmus wie die andern haben müssten (s. 157), gibt er den versen des King Horn vier oder (bei klingendem ausgange) drei hebungen, unterscheidet daneben aber auch noch eine anzahl verse, die nach alter weise mit zwei hebungen zu lesen seien. Also - finden sich in einem gedichte von 1500 zeilen zweifach, dreifach und vierfach gehobene verse gleichberechtigt neben einander. Bei Lazamon musste 'im zusammenhange' derselbe rhythmus herrschen, hier scheint das nicht nötig. Indessen den me. dichtern passieren noch unbegreiflichere dinge.

Da gibt es mehrere gedichte, die nicht bloss den septenar und den alexandriner, sondern beide fremde metren und die alte langzeile neben einander aufweisen und also metren, die nach Sch. vier, sechs und sieben gehobene silben haben, ohne jedes bedenken gleichzeitig verwenden. So ist in dem gedichte ' $O n$ god Ureisun of ure Lefdi' (Old Engl. Hom., I. Ser. 191 ff.) nach Sch. viermal gehoben die zeile Cristes milde móder, seýnte Márie. Dass -ie in Marie reimt, tut nichts zur sache, die alliteration übertönt hier den endreim; das nachdrücklich vorangestellte Cristes steht im auftakt. Vierfach gehoben ist ferner die zeile:

mi lif and mi tohópe min hé́le mid iwisse.

Hier übersieht Sch., dass to in der nominalkomposition den ton haben muss. Man vgl. hierzu ein s. 175 von Sch. gegebenes beispiel, wo bei der betonung tóname die zwei hebungen herauskommen. 
Daneben finden sich nun in diesem gedichte auch noch zeilen, die nach Sch. nur in einer der beiden vershälften 'durchsus nur zwei hebungen' haben und somit ein richtiges metrisches zwittergebilde darstellen. Sch. führt deren s. 165 zehn auf, sie sind aber viel zahlreicher. Ich brauche kaum zu versichern, dass für uns halbzeilen wie ne wéopèn ne múrnèn, pi veir tó ¿seónnè, mid gúldène chéllè etc. regelmässig vier takte haben. Nur so entgehen wir auch der gefahr, dieselbe zeile entweder als langzeile mit vier, oder als alexandriner nit sechs, oder als septenar mit sieben hebungen zu lesen, in der sich Sch. ^. 167 befindet. Die zeile:

vór he néver ne beớo seád pi veír tó iseónnè

crlaubt für uns nur eine auffassung. Sch. ist allerdings ge1ötigt, s. 167 (und an andern stellen) darauf hinzuweisen, wie durch accentuation höher betonter senkungen (so ist an dieser stelle statt 'hebungen' zu lesen), aus einem zweihebigen ${ }^{1}$ ein riertaktiger vers entstehen konnte.

So kommen wir denn praktisch einander näher. Denn für nich ist es nicht zweifelhaft, dass diese nach Sch.'s theorie so rerschiedenen metra alle nach denselben gesetzen gebaut sind Ind als gleichartig empfunden wurden. Was zunächst den tetraneter anlangt, so ist er im grunde genommen weiter nichts als eine strenger gebaute langzeile, mit stumpfen ausgange vor

1 Es ist mir nicht entgangen, dass Schipper unterscheidet zwischen 'gleichhebigen' und 'gleichtaktigen' versen; dass ' $z$ weihebige' verse $z$. b. ron 'zweitaktigen' sehr verschieden sind. Ich vermag nur nicht recht 'a sehen, worin diese verschiedenheit besteht. Hier wie dort haben wir wei gehobene silben, und diese sind das wesentliche im verse. Der interschied wird also, bestimmt wird das nirgends gesagt, nur darin jestehen, dass in versen der ersteren art die senkung kein bestimmtes naass hat, während sie in solchen der zweiten art im allgemeinen nur sinsilbig sein darf. Freiheiten in der behandlung der senkung in fremlen metren fälirt Sch. auf germanische licenz zurïck s. 109. Dass diese teiheiten sich in gewissen grenzen bewegen, von denen sich das nach unserer theorie behandelte germanische metrum nicht entfernt, wïhrend lie nach Sch.'s theorie gemessene langzeile jeder schranke entbehrt, ist sch. entgangen. Auch hat er es versäumt, über den charakter der senkung in jener fremden metren genaue untersuchungen anzustellen Ind die eventuellen ergebnisse dieser untersuchung auf die german. angzeile anzuwenden. Freilich wiirde alsdann dic zweibebungstheorie sicht einen moment stand gẹalten haben. 
der cäsur und klingendem ausgang am schlusse. Ich glaube sogar, dass man die einführung dieses nicht gerade häufig vorkommenden mittellateinischen metrums in das Englische hauptsächlich seiner tibereinstimmung mit dei germanischen langzeile zu verdanken hat. Ich stehe darum auch nicht an, die ansicht Jessen's, dass Orm, indem er am schlusse seines verses nur tonfähige endungen verwendete, auch das zweite glied des septenars als ein eigentlich viertaktiges betrachtete, prof. Schipper gegenüber wider aufzunehmen. Damit soll nun aber nicht gesagt sein, dass wir nun wirklich betonen müssten kindé, trówwpé, und die endsilbe bis zu einem vollen takte zu dehnen hätten. Der takt wird gefüllt durch die am schlusse des verses nach der tönenden endung eintretende pause. Wenn ich in meinen Untersuchungen zu King Horn s. 48 sage, dass bei klingendem ausgange die vierte hebung auf der klingenden silbe ruht, so ist dies, wenigstens in dieser so späten zeit, nicht anders zu verstehen. Hier sowol wie bei Orm wird erst durch die pause die klingend ausgehende zeile resp. halbzeile der vierfach gehobenen rhythmisch gleichwertig, wie in dem bekannten gleichartigen, im Me. häufig zur strophenbildung verwendeten, im Deutschen noch heute volkstümlichen metrum, das noch jüngst ein österreichischer dichter mit glïck verwendet hat:

Anhében lásst uns állzusańm'

Ein liéd von stárkem klángè.

Dem entspricht es denn durchaus, dass in andern nachbildungen desselben metrums in der ersten vershälfte neben vier hebungen mit stummpfem schluss, ganz german. regel entsprechend, aúch drei hebungen mit klingendem ausgange begegnen. Es dies z. b. der fall mit dem gedichte 'The Woman of Samaria' (An Old English Misc. s. 84 ff.). Hier finden sich neben einander die halbzeilen:

$F_{0}$ ihésu crist an eórpe wias, A'lle heo beóp on bóke ivv'ýten; A luitel tefor pe týmè, peo wýmmon him onswéredè.

Hiertber genaueres im anhang no. 2. Nach Sch.'s ansicht sind hier septenare und alexandriner gemischt. Damit beschreibt er das metrum, ohne indessen die vermischung zu erklären. Eine hinreichende erklärung gibt nür die annahme, dass auch bei den nachahmern fremder metren die gesetze des nationalen verses, der alten langzeile, noch fortwirkten. Ganz unberechtigt ist darum Sch.'s versuch s. 120, durch beseitigung einer 
hebung in der" ersten hälfte von z. 65 dieses gedichtes ein reimpaar von angeblich gleichartigen langzeilen (von je drei hebungen in der ersten halbzeile) zu gewinnen. Gerade durch diese änderung würde die ungleichbeit in den ersten vershälften erst herbeigefuhrt werden.

Auf .romanischen einfluss aber, und zwar den des alexandriners, ist es zurückzuführen, wenn in der zweiten hälfte dieser langzeile neben dem gewöhnlichen klingenden ausgange auch stumpfer ausgang sich findet, so dass wir tatsächlich nur drei hebungen haben und die zweite vershälfte gegen die erste um eine hebung gekürzt erscheint. Eine erklärung für diese kürzung ist nicht schwer zu geben. Gieng die zweite hälfte der langzeile, wie es beim tetrameter der fall war, regelmässig klingend aus, so musste dies bei einer längeren folge von derartigen zeilen gegenüber der ersten hälfte den eindruck einer kürzung um etwa zwei drittel takte hervorrufen. Von da wurde die kürzung bis zu einem vollen takte kaum noch empfunden. So erhielt man das schema der Nibelungenzeile (in den drei ersten versen der strophe), welches sich bei drei hebungen klingend in der ersten vershälfte mit einer der häufigsten formen des alexandriners deckt und in deutlicher zweiteilung im Deutschen noch heute eines der beliebtesten lyrischen maasse bildet und u. a. von Friedrich von Sallet gerne verwendet wurde, z. b.:

Ein eisenblanker ritter,

Geharnischt ganz und gar.

Dieses metrum nun, welches in seiner ersten hälfte vier hebungen mit stumpfem (ein- oder zweisilbigem) oder drei bebungen mit klingendem ausgange, in der zweiten bälfte dagegen drei hebungen entweder mit klingendem oder mit stumpfem ausgange zeigt, und welches, von dieser letztgenannten form abgesehen, durchaus das maass der alten langzeile bildet, ist in vielen gedichten dieser periode verwendet. ${ }^{1}$ In dem schon genannten 'On god Ureisun of ure Lefdi' (Old Engl. Hom., I. Ser. s. $191 \mathrm{ff}$.) haben wir als erste vershälften:

And gód pu irt and góde leof, pú ert briht and blisfül, Biuóren bine leófe suine; Swride wél ham likèd etc.

Die zweite halbzeile hat meistens drei hebungen mit klingenschlusse.

1 Bereits in der Samariterin begegnet ein reimpaar mit stumpfem 
dem ausgang, daneben auch noch öfters viè hebungen mit stumpfem schluss, eine form, die nur auf die alte langzeile zurïckgehen kann. Auch finden sich halbzeilen von nur drei hebungen: 145 pet pe deouel hatied me, 146 pet ich wurdie pe. Näheres im anhang no. 1.

Durchaus in dem oben beschriebenen metrum ist gedichtet 'A lutel soth Sermun' (An Old Engl. Misc. s. 186 ff., s. anhang no. 3) mit zweiten vershälften:

weindit to hille, and speker of derne hiue, tó pe hilididy, und, das umfangreichste, 'The Passioun of our Lord' (Old Engl. Misc. s. $37 \mathrm{ff}$.). Auch hier ist die erste halbzeile noch durcbaus als viertaktig im alten sinne anzusehen. Alle ersten halbzeilen mit stumpfem ausgange haben vier hebungen, vgl. anhang no. 4. In dem von Sch. s. $118 \mathrm{f}$. ausgehobenen abschnitte aus diesem gedichte ist darum z. 23 zu betonen ofspruing (dieselbe halbzeile begegnet in der Moral Ode 207 und in Doomsday 53), 34 A'dám; 36 fehlt die senkung zwischen nouht und pat; 37 fehlt auftakt und erste senkung; 101 ist zu betonen po seýdc úre lóuerd crist (nicht bo seýdẹ ure lóuerd c.). Ferner findet sich dieses metrum in einer anzahl strophischer gedichte, die zum teil schon genannt sind (s. oben) und im anhange eingehend erörtert werden.

Wir haben also nicht nur erwiesen, dass Schipper's versuch, die anwendbarkeit der Lachmann'schen betonungsgesetze auf das Englische zu leugnen und damit eine stütze für die zweihebungstheorie $z u$ gewinnen, misglïckt ist; wir gelangten durch unsere betrachtungen auch zu einem neuen argumente für unsere theorie der ae. langzeile, wonach jede hälfte derselben vier hebungen hat. Denn wenn noch im Me. mit halbzeilen, die unleugbar vierfach gehoben sind, wie z. b. aus ' $O n$ god Ureisun of ure Lefdi' 50 Hám pet swinked dei and niht, sich solche mischen, die nur unter anwendung der Lachmann'schen betonungsgesetze das gleiche maass erhalten, wie 13 A'lle cristène mén, oder nur durch annahme der fortdauernden wirksamkeit der alten versprinzipien mit jenen rhythmische gleichheit gewinnen, wie 72 Siggeঠ ánd singèঠ, so müssen wir darin eine crw ïnschte, wenn auch nicht unerwartete bestätigung der richtigkeit unseres standpunktes in beiden beziehungen erkennen.

Wenn Schipper, anm. zu s. 141, aus Siever's aufsatz 'Zur Accent- und Lautlehre der germanischen Sprachen' in Paul und 
Braune's Beiträgen IV, $522 \mathrm{ff}$. den schluss zieht, dass Sievers hinsichtlich der endungsvokale zweisilbiger wörter im Ahd. und Mhd. zu einem dem seinen analogen resultate komme, so weiss icb nicht, auf welche stelle jenes aufsatzes sich Sch. gründet. Meines erachtens dienen Siever's resultate dazu, die Lachmann'schen regeln nur zu bestätigen. Wenn aus goth. nasida ae. nerede, mhd. néreiè, aus haúsida aber ae. hŷrde, mhd. hô'rtì wurde, so beruht die elision im letzteren falle doch wol darauf, dass nach dem sprachgesetz dem auf die lange stammsilbe folgenden $e$ der ton gebührte; nun schien aber die flexionssilbe wichtiger zur formbildung, eine betonung hốretè jedoch war sprachwidrig, folglich musste der mittlere vokal ausfallen, damit der wichtigere vokal an die richtige tonstelle trat. Nicht anders verfährt z. b. noch Orm. Formen wie cwemmde, demmde (p. prät. pl.); hallghe, little; deoffles, posstless, tacness etc. beruhen auf demselben prinzip. Dass er andrerseits auch ein $e$ auswirft, um zweisilbige formen zu erhalten, wie heoffne, seffne, fremmde etc. widerspricht dem nicht.

Nirgends aber macht Sievers in der angezogenen schrift gegen Lachmann's betonung des mhd. verses opposition. Im gegenteil, er spricht a. a. 0 . s. 528 ausdriticklich von der 'ictusfähigkeit der $e$ in hôrle, blinde im gegensatz zu dem von tage'. Er will nur in der prosa solche silben als unbetont betrachtet wissen. Es wird ihm aber sicher nicht einfallen, z. b. im Tristan nach Sch.'s weise betonen zu wollen: Von ríche ze ríche, Von lánde ze lánde, oder im Iwein den versen: Mîn hêr Gâwein, Hie slac dầ slich, Ir nôt uberwant, Den lop unde den prîs ete. die vier hebungen zu verweigern. Sind nun verse aus dem King Horn wie: Hi wenden to wisse, His feren bu wise, for to bihelde, And al quic hem fle ${ }^{1}$ von den aufgefiihrten beispielen aus den besten dichtern des Mittelhochdeutschen irgendwie verschieden?

Auf grund der vorangegangenen prinzipiellen auseinandersetzungen bleibt mir nur noch übrig, die auffassung, die Sch.

1 Ich benutze diesen anlass, um auf ein versehen in der einleitung zu meiner ausgabe des King Horn hinzuweisen. In dem abschnitt über die metrik sind einige beispiele, die unter II, 2 gehörten, unter I, 2 geraten. Gleichzeitig bitte ich auf s. XII, z. 13 v. u. statt 'für ae. $\zeta$ und' zu lesen 'für ae. $g$ in inlaut nach dunklem vokal und nach konsonanten und'. 
dem metrum des King Horn gibt, im einzelnen zu widerlegen und meine ausgabe, die Sch. noch nicht zur hand hatte, zu rechtfertigen.

Nach der bisher unter deutschen philologen ziemlich allgemein giltigen ansicht besteht das reimpaar des King Horn ganz wie das mittelhochd. kurze reimpaar aus versen, die bei klingendem ausgange drei, bei stumpfem (ein- oder zweisilbigem) ausgange vier gehobene silben aufweisen (beispiele: A'lle beón he blibè; A'lle swipe faire gúmes, O'fte hádde Hórn beo wó), , wobei in zeilen der ersten art die vierte hebung auf der klingenden silbe ruht. Sch. aber findet im K. H. zweimal, dreimal und viermal gehobene verse und die letzte silbe, einerlei ob mit stummem oder tonlosem $e$, ist nicht in anschlag zu bringen. In betreff des letzteren punktes beruft er sich auf den im 6. kapitel seines buches angeblich geführten beweis der absoluten hebungsunfähigkeit unbetonter endsilben, den wir oben zurückgewiesen haben.

Neben versen von drei hebungen mit klingendem ausgange begegnet aber nach Sch. (s. $182 \mathrm{f}$.) auch eine nicht unbeträchtliche anzabl von versen mit drei hebungen und stumpfem ausgang. Als solche, die möglicherweise mit drei hebungen zu skandieren seien, werden aufgeführt:

Z. $7 \mathrm{f}$. Godhild het his quen

fairer ne mizte non ben.

Während Sch. für alle von ihm bis dahin besprochenen fremden metren, den septenar, das rom. kurze reimpaar, den alexandriner, die möglichkeit des fehlens der senkung nach deutschem prinzip zugesteht, scheint er diese möglichkeit in rein deutschen metren nicht zugeben zu wollen. Dem worte Godhild zwei hebungen zu geben und danach die senkung fehlen zu lassen hat doch gar keine schwierigkeit. Auch in der folgenden zeile fehlt einfach eine senkung. So erledigen sich nun fast alle weiter aufgeführten zeilen:

$33 \mathrm{f}$. (ich citiere nach meiner ausgabe) ist zu lesen:

Múrrì pe góde kíng ród on his pléing.

$87 \mathrm{f}$. páỳns him wólde slén

óper ál quíc flén (nachdrücklich, ähnlich)

1394 and ál quíc hém flé.

$139 \mathrm{f}$. pánne spák him chíld Hórn

In Súddène he wás iboŕn (oder I'n Sudénne etc). 
Wie Sch. ïberhaupt hier eine schwierigkeit finden konnte, bcgreife ich nicht.

$163 \mathrm{f}$. hé him spác to Hórn chíld wordès swípe ( 0 wel swipe, $C$ pat were) míld.

Z. $525 \mathrm{f}$. bereitet keine schwierigkeit. In 519 habe ich aus $\mathrm{H}$ ein die senkung füllendes $b i$ aufgenommen. $1143 \mathrm{f}$. entspricht die betonung gálún : glótún einer von Lachmann aufgestellten regel (lied von K. H., einl. XVIII; in 1143 ist vielleicht besser one statt $a \mathrm{zu}$ lesen, wie ich in den nachträgen vorschlage). Einige schwierigkeit macht nur z. 453 (in 454 ist dúbbing ohne bedenken). Ich habe $\mathrm{C}$ belassen und betone: tó mi lórd pe king. Leicht wäre die änderung lóuèrd (nach $\mathrm{H}$ ) oder kingè (nach 0 , dann in der folgenden zeile dubbinge). Jedenfalls haben wir auch hier keine 'drei' hebungen. Auf keine weise nun, 'wenn wir nicht der natürlichen, sinngemässen betonung arge gewalt antun wollen', mit vier, sondern nur mit drei hebungen können nach Sch. gelesen werden:

$95 \mathrm{f}$. pu art gret and strong

fair and euene long;

wo ich jedesmal den drei ersten worten je eine hebung gebe. Uebrigens wäre es leicht gewesen, nach $\mathrm{OH}$ die senkung zu füllen. Fur unbedenklich halte ich auch die zeilen:

$267 \mathrm{f}$. In héorte héo hádde wó

And pús híre bipózte pó.

Auch hier futlen $\mathrm{OH}$ die senkung.

Für 335 f. schlage ich im nachtrag vor, Horne: unorne zu lesen. $363 \mathrm{f}$. betone ich:

pú schalt béo dúbbed knízt

A're (ausg. Er pan) cóme séue nízt.

Z. 92 wïrden $\mathrm{OH}$ die füllung der senkung erlauben. 495 betone ich hit nére nózt fórlórn. Die stelle ist übrigens unsicher. 496 ist unbedenklich. $506 \mathrm{kann}$ auch in der fassung von C (himsélf) keinen anstoss geben. $895 \mathrm{f}$. stehen nur in $\mathrm{C}$; ich lese:

ánd pát his fáder slóz

to hím his swérd he dróz.

Es liegt ein besonderer nachdruck auf diesen zeilen. $1141 \mathrm{f}$. folge ich $\mathrm{OH}$, desgleichen $1327 \mathrm{f}$. (nicht aus metrischen grïnden), ebenso $1375 \mathrm{f}$. (gleichfalls nicht aus metrischen grtinden).

So bliebe nur ein reimpaar, wo alle hss. die annahme von nur drei hebungen erlauben, nämlich $601 \mathrm{f}$. Die fälle indessen, wo die präposition den hochton erhält, wie auch 
Lachmann für das Mhd. zugibt, sind nicht selten. Auch and steht öfters in der hebung. Ich nehme daher gar keinen anstand, auch hier zu betonen:

\section{Léue át híre he nám \\ Ańd ínto hálle cám}

(biatus ist erlaubt, einl. XVlII f.). Sch. hätte also das vorkommen von versen mit nur drei hebungen 'doch nicht hinlänglich erwiesen'.

Weiter sucht Schipper darzutun, dass auch verse mit vier hebungen und klingendem ausgang vorkommen, und dies mit mehr berechtigung. Derartige verse kommen ja 'am ende der abschnitte des sinnes', oder wo die erzählung ruht, auch im Mhd. vor (vgl. Lachmann zu Iwein 722). Wir hätten also wenig grund, dieselben für den K. H. zu leugnen. Vier hebungen mit stummer endsilbe sind natürlich erlaubt. Irrtümlicher weise führt Sch. als beispiel hierfür unter andern $621 \mathrm{f}$. (637 f. meiner ausgabe) heued: bireued auf, wo langer stammvokal vorliegt; auch $1355 \mathrm{f}$. (1379 f. meiner ausg.) ist nicht ganz passend.

Von den versen mit klingendem ausgange wurden in meiner ausgabe nach 0 oder $H$ oder nach beiden berichtigt $661 \mathrm{f}$. und 907 f.; 327 f. gibt keinen anstoss, ebenso wenig 1153 f. 1361 steht Horn im auftakt, $u t$ war zu tilgen, vielleicht ist auch noch die umstellung nach $\mathrm{OH}$ vorzunehmen; 1362 steht twelf im auftakt, für felazes schlage ich in den nachträgen childen vor; 89 ist $\mathrm{zu}$ betonen:

\section{3if hís fairnésse nere}

(vgl. einl. s. XVIII); in 90 war alle nach $\mathrm{OH}$ zu tilgen.

Z. 1363 ist among in den auftakt zu setzen, vielleicht als mong nach 0 ; mang begegnet $z$. b. auch bei Orm in auftakt. In der folgenden zeile war leue nach 0 zu streichen.

Wirklich vier hebungen könnte man annehmen in $583 \mathrm{f}$. Jedoch hatten $584 \mathrm{OH}$ regelmässig drei hebungen klingend. So suchte ich auch 583 nach $\mathrm{H}$ mit unterstiatzung von $\mathrm{O} \mathrm{zu}$ bessern. Immerhin bleibt nis non schwer für den auftakt. Vielleicht ist zu lesen: nis nón betre (statt betere) únder sunne (man vgl. 'A Luue Ron', Old Engl. Misc. s. 88, 154: Nis nón betere únder heóuene grúnde). Die schwierigkeiten, die $1389 \mathrm{f}$. bieten, werden in den anmmerkungen zu meiner ausgabe besprochen. Die möglichkeit, auch hier mit vier hebungen und klingendem schlusse zu lesen, will ich zugeben, desgleichen 
ftir das reimpaar $1433 \mathrm{f}$, dessen erste zeile in allen hss. überfüllt ist (ich habe mit widerstreben den art. vor day getilgt); 1434 hat nach $\mathrm{H}$ nur drei hebungen (in der anm. zu dieser stelle ist statt $\mathrm{OH}$ vielmehr $\mathrm{OC}$ zu lesen).

Es reimen nach Sch. s. 184 zweisilbige worte mit kurzer auf solche mit langer stammsilbe, nicht bloss in zeilen von drei hebungen und weiblichem ausgange, sondern auch in solchen von vier hebungen. Als einziges beispiel wird $297 \mathrm{f}$. genannt. Dieses reimpaar ist aber in allen hss. variiert (s. m. ausg. und anm.). Ich habe $\mathrm{C}$ im wesentlichen belassen, indem ich Apelbrus (besser Aylbrus) nur einen hochton gebe und im folgenden verse nach verwandlung des into in to $(\mathrm{OH})$ zweisilbigen auftakt annehme. zede hat langen vokal, wie auch bei Orm (s. oben). Schwieriger ist z. 251 (von Scb. nicht aufgeführt) stede (ac. stede, styde) im reim auf drede (ae. drêd). Ich nehme auch hier verlängerung des vokals an und streiche mit 0 das opere in $\mathrm{C}$. Uebrigens scheint auch hier tiefere verderbniss zu herrschen.

Unbedingt mit langem vokal und demnach klingenden ausgang sind anzusetzen worte mit ursprünglichem $\breve{a}$ in offener silbe, im reime auf ae. $\hat{e}$, K. H. $a$ mit dem laute $c^{a}$, s. lied von K. H., einl. s. VI. ${ }^{1}$ Dass aber gar he auf deic (mit verstummter endsilbe) oder beon auf quene reimen sollte (s. Sch. a. a. o. s. 184), kann ich nicht glauben und habe auch vergeblich in Schipper's buche unter den abschnitten über den reim nach analogieen geforscht.

Es ergibt sich also aus unserer betrachtung mit sicherheit, dass verse von vier hebungen und klingendem ausgange, wenn uberhaupt, nur in ganz beschränktem maasse in unserem liede für zulässig erachtet werden können, wofür es ja im Mhd. an analogieen nicht fehlt. Die tonfähigkeit der unbetonten endsilbe wird dadurch in keinem falle berührt.

Ausserdem will nun Sch., wie wir oben schon hervorhoben, s. 190 seines buches im K. H. auch noch solche zeilen erkennen,

1 Die hs. C reint fairhede : makede, makede : verade. Ich dachte zuerst daran, dass hier nur die tonlosen endsilben reimten, wie mhd. Hagene : degene, ebenso in ofherde : andswarede, ferde: hurede. Doch war in allen diesen fällen klingender reim leicht herzustellen, meist auch in anderen hss. iiberliefert, so dass ich $\mathrm{C}$ fallen liess. Vgl. jedoch Einenkel a. a. o. s. 45. 
in denen die ' $z$ wei hebungen des alten halbverses .... noch scharf und, ohne die möglichkeit oder wahrscheinlichkeit einer dritten hebung zuzulassen, hervortreten'. Die aufgeführten zeilen sind durchaus den gesetzen des deutschen verses entsprechend gebaut und zu betonen:

1399 f. hi slózen ánd fúztèn

pe nízt ánd pe úztèn.

123 hi wéndèn to wíssè (oder: hi wénden tó wíssè).

215 fram kíngè to kíngè (oder: fram kínge tó kíngè).

293 in Hórnès ilíkè.

1277 hi rúngè pe béllè.

Nach Schipper weist also das lied von King Horn verse von zwei, von drei (stumpf und klingend) und vier (stumpf und klingend) hebungen auf. Damit wäre die absolute formlosigkeit als prinzip proklamiert, die wir oben schon als die konsequenz der von Schipper vertretenen theorie hingestellt haben.

\section{An hang.}

Um ein möglichst genaues bild der besprochenen sogenannten me. langzeile in ihren frühesten erscheinungen zu geben, folgt, unter den im voraufgehenden aufsatze dargelegten gesichtspunkten, eine zusammenstellung aller halbzeilen aus den ersten und bedeutendsten in diesem metrum abgefassten gedichten. Es wird dies einerseits die nachprïfung meiner auffassung dieses metrums erleichtern, andererseits vielleicht auch zu weiterer, systematischer forschung auf diesem gebiete anregen.

1. In dem gedichte 'On god Ureisun of ure Lefdi', Old Engl. Hom., I. Ser., XX, s. $191 \mathrm{ff}$., haben wir in der ersten vershälfte

1. Vier hebungen mit stumpfem ausgang und zwar a) einsilbig in den zeilen 4. 5.7 (senkung fehlt). 8 (s. f.). 11 (zwei senkungen fehlen). 12. 13. 11.15 (alesẹd). 19. 20. 24. 25 (s. f.). 30.31 (blissè ne méi). 31. 36. 38 (néuir ne mei). 40 (s. f.). 41. 42. 46. 50. 51 (s. f.). 54. 57. 61. 62.63. 68. 70. 73. 78. 80. 81. 82. 84. (ville is). 85. 88. 93. 96. 98. 99. 103. 109. 113. 114. 115. 116. 121. 124. 125. 129. 131. 132. 133. 134. 136. 149. 150. 157. 159. 160. 164. 165. 166. 167. 170, zusammen 68 ; b) zweisilbig ohne möglichkeit der verschleifung, z. 6. 21 (maidéne). 23 (vuimmòn). 26.53 (rǒse?). 67. 76. 106. 107. 108. 117. 127 (ínwine). 130 (wuirchipe). 137 (feier). 141. 146 (?). 151. 169 (miinuch), zusammen 18; c) zweisilbig mit möglichkeit der verschleifung, z. 18. 71 (?). 95 (luue ne). 112. 126(?). 163 (licáme). Summe aller stumpfen ausgänge 92.

2. Drei hebungen mit klingendem ausgang: a) ohne möglichkeit der verschleifung, z. 1. 2. 9. 17. 27. 28. 29. 33. 35. 37. 39. 43.44 (wéopèn ne). 45. 47. 48. 49. 52. 55. 56 (pet ham in der senkung?). 58. 59. 65. 67. 69. 72. 74. 75(?). 77. 83. 86. 87. 89. 91. 92. 93. 100. 102. 104. 105.110. 111. 118. 119. 122. 123. 139. 140. 142. 143. 144. 145. 147. 153. 157. 168. 
171 , zusammen 57 ; b) nöglicherweise verschliffen, $\alpha$ ) vor vokalen, z. 3 . 10. 32. 60. 79. 97. 120. 128 (drāwe?). 152. 155. 161. 162, $\beta$ ) vor $h$ z. 90, $\gamma)$ mit der folgenden senkung (to, ne, bi-) z. 16. 22. 139. 148. 153, zusammen 18. Summe aller klingenden ausgänge 75 .

3. Vier hebungen, a) verschleifbar, z. 101 . $64(?) .156$; b) unverschleifbar 135 (lihtlichẹ).

4. Möglicherweise mit drei hebungen und stumpfem ausgange sind zu lesen: z. 71 (Al englene were). 126 (Vor is holie nome). 128 (Ne pet he me drawe, wo vielleicht langer vokal anzunehmen, denn 141 reimt todrawe auf fawe, zu ae. f(êznian). Doch kann man hier iiberall auch vier gehobene silben annehmen, ohne der deutschen wortbetonung zwang anzutun.

Die summe der stumpfen ausgänge übertrifft die der klingenden etwa um ein viertel.

In der zweiten vershälfte haben wir

1. drei hebungen mit stumpfem ausgang z. 55(?). 56(?). 129. 130. 145. 146. 166. 167. Die zeilen 71. 99. 122. 157 sind um deswillen unsicher, weil die entsprechenden reimzeilen besser mit vier hebungen gelesen werden.

2. Vier hebungen mit stumpfem ausgang, a) einsilbig, z. 72. 100. 121. 158 ; b) zweisilbig, z. 13. 14. 41. 42. 65. 66. 67. 65. 119. 120. 125. 126. 143.144 .

3. Alle übrigen zeilen haben drei hebungen mit klingenden ausgang. Z. 73 f. reimt ore : uorlore[n].

2. Das gedicht 'The Woman of Samaria', Old lingl. Misc. s. $84 \mathrm{ff}$. zeigt in der ersten vershälfte

1. vier hebungen stumpf, a) einsilbig (alle tieftonigen ausgänge mitgerechnet), z. 1. 6. 11. 14. 16. 17. 19. 20. 21. 22. 24 (péróf). 25. 28.29. 33. 35. 37. 38. 43. 45. 46. 47. 48. 49. 52. 55. 56. 57. 58. 59. 61. 64. 65. 69. i2 (buruh, bureh). 73. 74. 76. 77; b) zweisilbig, $\alpha$ ) unverschliffen z. 2. 3. 4. 8. 15 (?). 23. 34. 35. 36. 60. 62.68 (iseye), $\beta$ ) verschleif bar auf vokale, z. 30. 31. 41. Summe der stumpfen ausgänge 54 .

2. Drei hebungen klingend, a) unverschliffen, z. 5. 12. 32. 39. 51. 53. 63. 66 ; b) verschleif bar, $\alpha$ ) auf vokale, z. 13. 18. 50. 54. 57. 67. 70. 71. $75, \beta)$ auf $h$, z. $27, \gamma$ ) auf die fulgende senkung z. 26. 40. 41. 44. Summe der klingenden ausgänge 21.

3. Vier hebungen klingend, unverschleifbar, z. 9 (bileuynge). 10 (Heo duden heore louerdes hestes, vielleicht dude heore?).

4. Möglicherweise haben drei hebungen z. 11 (Al só heo wérèn agón). 15 (A'se heo wes ér iwined). 43 (And álle pilke pát beoop). 49 (paih he nóuper ne béo). 72 (And írnen uit of pe bireuh). 74 (pó byléuède pat fólk).

Die summe der stumpfen ausgänge iibertrifft die der klingenden un mehr als das doppelte.

Die zweite halbzeile hat drei halbzeilen klingend mit ausnahme von z. 55 f. (Messyas : was).

3. Das gedicht 'A lutel soth Sermun', Old Engl. Misc. XXV, s. $186 \mathrm{ff}$. besteht zunächst aus einer einleitung von acht langzeilen, deren crste 
hälfte vier hebungen stumpf zeigt in z. 1. 9. 13. 15 (nach halbzeilen gezählt), zweisilbig z. 7; drei hebungen klingend haben z. 3. 5. 11. Die zweite hälfte hat drei hebungen stumpf in z. 2. 4. 14. 16; klingend 6. 8 . 10. 12. Dann folgen vier kurze reimpaare romanischer art. In dem eigentlichen gedichte hat die erste halbzeile

1. vier hebungen stumpf, a) einsilbig, z. 33. 37. 53. 57. 63. 65. 71 . 73. 79 (míd him). 83. 95. 9i; b) zweisilbig, unverschliffen, z. 25. 27. 35. 45. 47. 55. 61. 89. 91, verschliffen 77. Summe der stumpfen ausgänge 22.

2. Drei hebungen klingend, unverschliffen, z. 29. 31. 39. 41. 43. 49. 51. 59. 69. 75. 81. 93. 99, verschleif bar 85. 87. Summe der klingenden ausgänge 15, die also von den stumpfen um ein drittel iibertroffen werden.

3. Vier hebungen mit klingendem ausgang zeigt z. 67 (At hom is hire pater noster).

Die zweite halbzeile hat

1. drei hebungen stumpf, a) einsilbig, z. 54. 56. 62(?). 64. 70. 72. 86. 88 ; b) zweisilbig, z. 42. 44. 50. 52. 58. 60. 74. 76. 78. 80. Summe der stumpfen ausgänge 18.

2. Drei hebungen klingend 26. 28. 30. 32. 34. 36. 35, 40. 46. 48. 66. 68. 52. 84 . 90. 92. 94. 96. 98. 100. Summe 20. Die zahl der stumpfen und der klingenden ausgänge ist also nahezu gleich.

4. In dem gedichte 'The Passioun of our Lord', Old Engl. Misc., s. $37 \mathrm{ff}$. haben wir in der ersten vershälfte

1. vier hebungen (alle tieftonigen silben am schlusse mitgerechnet) stumpf, a) einsilbig, z. 7. 10. 12. 13. 16. 19. 21. 23. 30. 31. 34. 36. 37. 38 . 41. 46. 47. 53. 54. 56. 57. 60. 62. 63. 65. 69. 71. 72. 74. 79. 80. 82. 88. 89 . 96. 101. 3. 5. 6. 8. 11. 18. 20. 21. 25. 28. 29. 31. 32. 33. 34. 47. 50. 52.53. 57. 61. 62. 67. 69. 72. 74. 75. 77. 78. 81. 89. 93. 96. 97. 99. 200. 3. 9. 12. 15. 19. 20. 24. 27. 28. 36. 37. 38. 39. 40. 45. 46. 49. 50. 51. 63. 64. 65.68. 71. 72. 74. 77. 78. 79. 82. 84. 86. 90. 91. 92. 95. 98. 303. 4. 6. 9. 14.15. 18. 21. 23. 31. 32. 33. 36. 44. 45. 47. 48. 50. 53. 57. 60. 65. 69. 72. 75. 7 7. 81. 82. 90. 91. 94. 95. 400. 3. 4. 7. 15. 20. 21. 23. 24. 28. 30. 31. 37. 39. 40. 42. 47. 49. 50. 51. 53. 54. 59. 61. 63. 67. 68. 70. 72. 73. 74. 77. 79. 83. 85. 91. 95. 501. 4. 5. 7. 8. 9. 10. 14. 16. 17. 20. 31. 34. 37. 40. 41. 42. 43. 44. 45. 47. 48. 49. ร1. 52. ร3. ร4. ร5. ร8. 63. 64. 65. 86. 67. 70. 72. 75. 76. 87. 97. 99. 601. 2. 5. 6. 7. 9. 13. 16. 17. 23. 27. 35 (?). 36. 45. 49. 59. 60 . 65. 68. 74. 79. 80. 88. 91. 94. 702. 703, zusammen 246 ; b) zweisilbig, a) unverschliffen, z. 1. 20. 39. 44. 49. 77. 124 (?). 187. 202. 214. 217. 257. 269. 280. 319. 392. 413. 488. 500. 506. 512. 525. 528. 546. 573. 594. 618. 621. 626. 652. 696. 697. 704. $705 ; \beta$ ) verschliffen auf vokale, z. 2. 58. 142. 146. 165. 171. 255. 328. 361. 378. 405. 406. 414. 513. 522. 536. 557. 559 . 586. 592. 600. 625. 693 , auf $h$, z.6.61. 235. 300. 670, anf die folgende senkung, z. 17. 59. 83. 266. 329. 338. 401. 476. 562. 611; zusammen 72 . Summe der stumpfen ansgänge 318 .

2. Drei hebungen klingend, a) unverschileifbar, z. 4. 5. 8. 14. 22. 25(?). 26. 28. 29 (?). 32. 40. 42. 48. 64. 70. 76. 85. 86. 91. 92. 93. 94. 97. 98. 110. 13. 14. 15. 16. 17. 19. 22. 26. 30. 36. 37. 38. 39(?). 43. 44. 45. 51 . 55. 60. 66. 68. 76. 79. 80. 82. 83. 84. 85. 86. 87 (?), 88. 90. 91. 94. 95. 201. 
4. 5. 6. 7. 8. 10. 11. 16. 21. 22. 32. 34(?). 52. 53. 54. 56. 59. 60. 61. 70. S1. 85. 87. 89. 97. 301. 5. 7. 10. 11. 13. 16. 22. 24. 25. 30. 35. 37. 40. 46. ว4. 59. 62. 64. 66. 73. 74. 76. 80. 83. 84. 85. 86. 99. 408. 11. 12. 18. 19. 22. 25. 26. 29. 32. 33. 36. 38. 41. 45. 46. 48. 52. 55. 60. 64. 65. 66. 69. 71. 78. 81. 84. 86. 5 7. 90. 96. 99. 503. 15. 19. 21. 23. 24. 26. 27. 30. 32.33. 35. 38. 39. 60. 61. 78. 79. 84. 89. 91. 95. 604. 14. 15. 20. 22. 24. 30.31. 32. 34. 38. 40. 41. 42. 43. 44. 46. 47. 48. 53. 55. 61. 63. 64. 67. 69. 71. 72. 73. 75. 77. 78. 83.85 . 86. 87. 89. 90.95. 99. 700, zusammen 213 ; b) verschleifbar, $\alpha$ ) anf vokale, z. 24. 27. 35. 43. 50. 51. 52. 66. 68. 73. 78. 84. 87. 99. 100. 7. 9. 12. 35. 40. 41. 49. 56. 59. 73. 92. 98. 226. 29. 30. 43. 58. 67. 73. 93. 94. 96. 99. 302. 8. 12. 39. 41. 42. 43. 49. 51. 52. 58. 63. 67. 68. 71. 89. 93. 97. 98. 416. 27. 34. 56. 57. 58. 62. 75. 80. 97. 511. 29. 60. 69. 77. S0. 81. 83(?). 96. 98. 603. 10. 19. 37. 39. 51. 54. 56. 57. 58. 62. 66, zusammen $89 ; \beta)$ auf $h$, z. 11. 33. 75. 81. 95. 164. 213. 25. 41. 42. 47. 75. 76. 320. 26. 27. 34. 8 7. 493. 94. 98. 571. 82. 88 . 90. 93. 650. 76. 81. 98, zusammen $30 ; \gamma$ ) auf die folgende senkung, z. 45. 67. 104. 27. 48. 54. 55. 63. 223. 31. 33. 44. 48. 83. 88. 317. 70. 79. 88. 410. 17. 35. 82. 92. 502. 18. S5. 608. 12. 28. 84 . 92 . 706, zusammen 33. Summe der klingenden ausgänge 365 .

Die summe der verschleif baren ausgïnge ist eine so beträchtliche, dass man nicht wol an einen zufall glauben kann, wenn ich auch, da sonst dreihebige erste halbzeilen nicht mit sicherheit nachweisbar sind, die verschleifung nicht wirklich vollzogen sehen möchte. Unter $\gamma$ ) liessen sich noch weitere fälle von 2. a) auffiihren. siebentel.

Die klingenden ausgïnge iibertreffen die stumpfen etwa um ein

3. Vier hebungen klingend, a) verschliffen auf vokale, z. 15. 18. 55 . 170. 262. 355. 409. 574. 629, auf $h$, z. 123. 396, anf die folgende senkung, z. 3. $90($ ?). 402 (?). 443 (?). 489 ; b) unverschleif bar, z. 9 (?). $280,335(?)$. $356($ ?). $361.556($ ?). 568. 633 .

Eine halbzeile mit sicher nur drei hebungen ist nicht nachzuweisen. Nangelhaft könnte erscheinen z. 551 And yef pat eche lif; ähnlich z. 505 . 507. 635. 674. 694. Vergleicht man jedoch halbzeilen wie 247. 248, wo wir, selbst um drei hebungen zu erhalten, betonen miissen: pis wé ihérde, po ouswérede, so haben auch die genannten zeilen nichts auffallendes. Z. 250 hio binuste pe dire bestätigt die regel, dass auf gehobenes tonloses $e$ wider eine schwache silbe als senkung folgen muss.

Die zweite halbzeile hat durchaus drei hebungen, und zwar stellen sich klingende und stumpfe ausgänge (bei letzteren die zweisilbigen, bei ersteren die dreisilbigen mit erster, kurzer, betonter silbe eingerechnet) an zahl ziemlich gleich, ohne jedoch gleichmïssig verteilt zu sein. Ursprünglich kurzer vokal in offener silbe scheint schon zum teil verlängert. Es reimt 375 inume : prisune; lang ist vielleicht auch $a$ in lane : dave (daye) 83. 325. 339. Zu bemerken ist der reim sone: to me 335 .

In der ersten vershälfte habe ich mit allem vorbehalt als lang, demnach mit klingendem ausgang, betrachtet: askapep 124, naked 234, quite 310, stanes 179, yete (adv.) 187. 583. Z. 609 reimt yct auf fet. Das wort louerd ist meistens, namentlich am ausgange der ersten vershälfte, 
als einsilbig anzusehen, so z. 111. 126. 129. 143. 148. 300. 347. 360. 423. 431. 564. 570. 685; genit. und dat. sind zweisilbig z. 456. 462. 560; 228. 596 (louerdes). Das wort Pylates hat doppelte betonung. Pýlatés am schlusse der halbzeile 323. 505. 507, Pilites meist im auftakt z. 325. 331. 339. 345. 347 etc. Ob auch Pilates z. 417 ?

5. Das gedicht 'On Serving Crist', Old. Engl. Misc. IX, s. $91 \mathrm{ff}$. (in ziemlicher verderbniss überliefert), zeigt in der ersten vershälfte

1. vier hebungen stumpf, einsilbig, z. 1 (séruè $w e$ ?). 2. 3. 4. 8. 9. 12. 16 (senkung tehlt). 17. 19. 22. 23. 24 (s. f.). 26 (s. f.). 34. 44. 46. 50. 52. 53. 57 (biscóp). 58. 63. 65. 66. 73. 78; zweisilbig, unverschliffen 40 . 55. 61 , verschleifbar 48 ; zusammen 31 .

2. Drei hebungen klingend, unverschliffen, z. 5.6 (senkung überfüllt). 7. 10. 11 (zweis. auftakt). 13. 14. 15. 18 (ïberfüllt). 20. 21. 27. 29. 31. 33. 35. 36. 37. 38 (iiberfiillt). 45. 48. 49. 51. 54. 56. 59 (läwe?). 60. 70. 71. 72. 74 . 77 , verschleifbar z. 25. 39. 47. 60. 62. 67. 69. 75. 76; zusammen 41.

3. Vier hebungen klingend, verschleif bar 7. 32, unverschleifbar z. 30 (?). 42. 43 . 64 .

4. Drei hebungen stumpf, z. 41 (?). 68(?).

Die zahl der klingenden ausgänge bei drei hebungen übertrifft die der stumpfen bei vier hebungen um ein viertel.

Die zweite vershälfte zeigt drei hebungen, wit stumpfem ausgange z. $1-8$. $25-32$. $51-56$. $65-78$, zusammen 36 , klingend z. 9-24. 33-42. 43-50. 57-62, zusammen 42. Das verhältniss ist also nahezu gleich. $\mathrm{Zn}$ bemerken ist die längere aufeinanderfolge desselben reimes. Ganz ähnlich ist das gedicht

6. 'Hwon holy chirche is onder uote', Old Engl. Misc. VII, s. 89. Die er'ste vershälfte hat

1. vier hebungen stumpf, einsilbig, z. 2. 5. 7. S. 9. 12. 15. 18. 21. 34, zweisilbig, unverschleifbar, z. 6 (würpsype). 10. 20. 33 (daye?). 35, verschleifbar z. 24, zusammen 16.

2. Drei hebungen klingend, unverschliffen, z. 1. 3. 4. 11. 13. 16. 22. 23. 26. 27. 28. 29. 30. 31. 32, verschleif bar, z. 14. 17. 19. 25. 36, zus. 20. Das verhältniss der stumpfen und der klingenden ausgänge ist also fast gleich.

Schwierigere betonungen sind z. 26 äl hire wéorrè $p, 27$ bispes ind clérekès, 28 kinges ánd eórlès. Die zweite vershälfte zeigt drei hebungen stumpf z. 1-6, klingend 7-10. 11-16. 17-20. 21-24. 25-28. 29.30. 31 . 32. 33-36. Hier überwiegen also die klingenden ausgänge bei weitem.

7. In dieser form ist $u$. a. anch noch abgefasst das kurze gedicht 'Judas', in Reliquiae Ant. ed. Wright, s. 144, dunach in Mïtzner's Sprachproben I, 1, s. 114.

Die erste vershälfte zeigt entweder vier hebungen stumpf, einsilbig, z. 1. 3. 5. 6. 11. 12. 13. 14 (Sóne só Júdás). 16. 17. 18. 22. 24. 26. 27, zweisilbig, z. 21. 23. 28 ; oder drei hebungen klingend, z. 2. 4. 7. 8. 9. 10. 15 . 19. 20. 25. 28. 30. 31.32. 33. Die zweite halbzeile zeigt durchgehends drei hebungen, entrveder mit stumpfem oder mit klingendem schluss. 
In den späteren in dieser langzeile gedichteten werken, den legenden, bei Robert of Gloncester etc. bleibt zwar die grundform noch bestehen. Die erste vershälfte zeigt auch hier meist noch entweder drei hebungen klingend, oder vier hebungen stumpf, daneben aber mehren sich die vershälften von vier hebungen mit klingendem ausgang. Die senkung wird freier behandelt; sio ist oft entschieden zweisilbig, so dass ein daktylischer rhythmus entsteht. Sie darf aber auch, ganz in der alten weise, fehlen. Eine umfassende untersuchung wiirde vielleicht recht dankenswerte resultate zu tage fördern.

8. Eine strophe von vier ganz regelmässigen tetrametern (vier hebungen stumpf, drei klingend) mit gleichem endreim steht unter dem titel 'Fortune' in An Old Engl. Misc. I, s. 86.

In vierzeiligen strophen sind weiter abgefasst:

9. 'A Prayer to our Lady', Old Engl. Misc. XXVII, s. 192 f. Hier haben wir in der ersten hälfte

1. vier hebungen stumpf, einsilbig, z. 10.11.12.13.14.15 16 (?). 18. 20. 31. 32. 34 (mi flésc.). 36. 38 (érènde). 39. 42. 44; zweisilbig, unverschleifbar, z. 9. 24. 43, zweisilbig verschleifbar, z. 14. 19; zusammen 22.

2. Drei hebungen klingend, unverschleif bar, z. 1. 2. 3 (?). 4. 5. 6. 7. 8. 21 (isuinehèd). 27. 29. 30. 35. 40; verschleifbar, z. 22. 23. 25. 28.33. 37. 41. Verlängert ist vielleicht $e$ in mete z. 26. Zusammen 22. Die zahl der stumpfen und der klingenden ausgänge ist also gleich. Die zweite hälfte geht noch stets klingend aus.

10. 'A Prayer to the Virgin', Old Engl. Misc. XXIX, s. 195 f. Die erste vershälfte-hat

1. vier hebungen stumpf, einsilbig, z. 1. 2. 4. 5. 8. 9 (mérci). 10. 11. 13. 15. 16. 17. 18. 19. 22. 25. 27. 28. 30. 31. 32. 34; zweisilbig, unverschliffen, z. 3. 6. 7 (make ne?). 20. 23. 24. 26. 33. 35, zusammen 31; wogegen die zahl der klingenden ausgänge verschwindet.

2. Drei hebungen klingend, z. 12. 14, 35(?).

3. Vier hebungen klingend, verschleifbar, z. 21 (houene ich), unverschleifbar, z. 29. 35(?).

Die zweite vershülfte geht mit ausnahme von str. 4 und 5 (drei hebungen stumpf) klingend aus.

11. Das gedicht 'Erhörung', Böddeker, Ae. Dichtungen des Ms. Harl. 2253. WL. XI, s. 172, hat in der ersten vershälfte ausschliesslich vier hebungen mit stumpfem ausgange, zweisilbig in z. 1. 11. 15 (verschliffen). 19. 34 ; vier hebungen klingend, z. 29 (scöle?). Der ansgang der zeile ist klingend mit ausnahme von str. 4 (drei hebungen stumpf).

12. Das gleiche maass hat WL. XII, Böddeker a. a. o. s. 174. Der ausgang der ersten halbzeile in z. 1. 2. 4 ist wol einsilbig zu lesen; verschleifung ist möglich in z. 10. Der schluss ist stets klingend.

13. Das gedicht 'Death', Old Engl. Misc. XXIII, s. $168 \mathrm{ff}$. (in zwei handschriften), in strophen von vier langzeilen mit gleichem endreim, zeigt in der ersten vershälfte (nach halbzeilen gezählt)

1. vier hebungen stumpf, a) einsilbig, z. 3. 7. 9. 13. 21. 2 i (pénni). 29. 33. 49. 63 (his fólc). 69. 73. 79. 85 (för pe). 97. 129. 139. 141 (in fü $r$ ). 
151 (pin his). 153 (senkung fehlt). 161 (of pe). 167. 183 (of pe oder senkung fehlt). 187(?). 199. 201(?). 207 (néauèr iseo). 209(?). 211. 213. 217. 225. 237 (him uit). 243 (him of). 247 (him ón). 257. 261 (pät wouih); b) zweisilbig, nnverschliffen, 7. 15. 23 (licome). 25. 41.61. 83 (baly, ae. belg). 109. 13 i (awciriède). 203 (ich schäl?), verschliffen z. 5 . 67(?), zusammen 48.

2. Drei hebungen klingend, a) unverschliffen, z. 1. 19. 31. 35. 37. 39. 45. 53. 57. 59. 65. 71. 75. 77. 81. 89. 91. 93 (str. XII). 99. 103. 105. 107. 111. 115. 117. 119 (str. XV). 121 (criste). 125. 127. 131. 133. 135.143. 145. 147. 149. 155. 157. 159. 163. 165. 169. 171. 173. 175 (str. XXII). 177. 179. 181. 189. 191. 193. 195. 205. 215. 219. 221. 223. 227. 229. 231. 235. 239 (?). 241. 243. 245. 251. 253. 255. (str. XXXII). 259, zusammen 68; b) verschleifbar, z. 11. 17. 43. 47. 51. 55. 87. 95. 101. 113. 123. 185. 197 . 233. 249. 263, zusammen 16. Summe aller klingenden ausgänge 83. Die klingenden ausgänge übertreffen demnach die stumpfen fast um das doppelte.

3. Möglicher weise nur drei hebungen stumpf haben z. 7. 9. 13. 21. 187. 201. 203. 207. 209. Doch sind notwendige betonungen der zweiten halbzeile wie 128 bópé bipauhte, 182 wórmès ifére, 188 pát pé bistod, oder (erste halbzeile) 45 pe sórie souilè derselben art wie die in den vorher aufgeführten zeilen verlangten.

Die zweite halbzeile geht einsilbig stumpf aus (drei hebungen) in den strophen VII. IX. X. XIX. XXIV. XXVI. XXVIII. XXIX. XXXII; zweisilbig in den strophen V. VIII. XXVII, zusammen 52. Alle übrigen ansgänge, zusammen 80 , sind klingend. Das verhältniss ist nahezu dasselbe wie in der ersten halbzeile.

14. Das gedicht 'Doounsday', Old Engl. Misc. XXII, s. 162 ff., gleichfalls in strophen von vier langzeilen mit gleichem endreim, hat in der ersten vershälfte

1. vier hebungen klingend, einsilbig, z. 1. 3 (senkung fehlt). 5. 9. 11. 13. 17. 19. 23 (?). 41. 47. 49. 53. 63. 67. 77. 81 ; zweisilbig, z. 7. 25.61 (suinnèn iwriten). 83. 85(?), zusammen 22.

2. Drei hebungen klingend, unverschliffen, z. 15. 27. 29. 31. 33 . 37. 43. 51. 59. 71. 7j. 79, verschleif bar, z. 21. 35. 39. 45. 55. 57. 65. 69 . 73. 87 , demnach in ziemlich bedeutendem prozentsatz. Summe der klingenden ausgänge 22. Die summe der klingenden und der stumpfen ansgänge ist also gleich.

Schwierigere betonnngen in den halbzeilen 23 (pát háued lútel idón). 47 (pát mákedè pe quéd). 61.85 (fór his hóli(e) nóme, dieselbe halbzeile in 'On god Ureisun of ure Lefdi' 126, mit der form holie). 77 (into berninde für); betonung in der zweiten hälfte z. 76 feóndès iférè.

Die zweite hälfte geht stumpf aus in strophe 4 ; strophe 5 reimen spere (sbst.), were (inf.), bere (inf.) auf gere (ae. garr). Die übrigen ausgänge (36) sind klingend bei regelmässig drei hebungen.

15. In strophen von meist - vier langzeilen ist abgefasst 'Dialog zwischen Leib und Seele', Büddeker a. a. o. s. 235, meist mit gleichem endreim, mit binnenreim (also reimordnung ab a babab) in strophe $I$. 
III. VI (nur z. 41. 45. 47). VII (nur z. 51. 52). VIII. XII (nur z. 59. 91. 93). XIII.

Die erste halbzeile zeigt

1. vier hebungen stumpf, a) einsilbig z. 1. 3. 5. 7. 13. 25 (?). 33. 35. 37. 43 (lýuedist). 49. 51. 53. 65. 71. 73. 79. 81. 83 (senkung fehlt). 87 . 89. 91. 93. 95. 117. 121. 125 (s. f.). 129. 143. 147. 149. 155 (s. f.). 167 (louerd einsilbig). 171 (senkung fehlt). 175. 179. 197. 199. 205. 217 (sóffrède). 233. 237. 239. 243. 245. 247, b) zweisilbig, 7. 55. 145.157 (verschleifbar). 165 (Cristès ycoren). 169. 177 (verschleifbar). 227 (verschl.), zusammen 53 .

2. Mit drei hebungen, klingend, a) unverschliffen, z. 9. 11. 15. 19. 27. 29. 31. 41. 45. 57. 59. 61. 63. 67. 69. 75. 77. 103. 109. 111 (s. f.). 115 (iiberfiillt). 131. 133. 135. 153. 161. 163. 173. 181. 183. 1S5. 187. 189. 201. 207. 209. 211. 215. 219. 221. 223. 225. 229. 231. 235. 241 ; b) verschleifbar, z. 17. 21. 23. 39. 47. 55. 97. 99. 101. 105. 107. 115. 123. 127. 159. 191. 193. 195. 203. 213. Summe der klingenden ausgänge 66. Dieselbe übertrifft die der stumpfen um ein fünftel. Als verlängert habe ich angesehen eten (inf.) $63 \mathrm{im}$ reime auf beten, prelen, und beren (inf.) 31. Verlïngert scheinen auch fare (inf.), bare (adj.), kare im reime auf mare (zweite balbzeile).

3. Vier hebnngen klingend, verschliffen, z. 113. 151, unverschleifbar z. 119 (?).

In der zweiten halbzeile überwiegt klingender ausgang, z. 2-8. 10-16. $18-24$. 50-56. 58-64. 66-72. 74-80. 82-88. 90-96. 114-120. 122-28. 130. 132. 144-50. 152-58. 160-66. 168-70. 176. 178. 204. 206. 220-28. 240. 242. 244-48, zusammen 74 .

Stumpf gehen aus, und zwar einsilbig, z. 26-32. 34-40. 98-104. 172-74. $180-86$. 158-94. 196-202. 208-14. 216. 218. 230-38; zweisilbig, z. 42-48: 106-112. 134. 136, zusammen 47 .

Nach z. 137 findet sich eine strophe von drei kurzen reimpaaren romanischer bildung.

16. Mit durchgehendem binnenreim ist gedichtet ' $\mathrm{T}$ 'he Duty of Christians', Old Engl. Misc. XXVII, s. 14l ff.

Reimordnung a b a b a a b.

b hat stets drei hebungen mit klingendem ausgange; a hat drei hebungen klingend nur in den strophen V. VI, sonst vier hebungen stumpf, doch reimt $\mathrm{z} .13$ on rode auf blod (god, edmod), 73 buten nyhte auf fiht (unriht, cnyht), 105 mylde : vilde auf ispild: child.

17. Ueberschritten wird das maass von vier hebungen in dem gleichfalls aus strophen von acht zeilen mit binnenreim bestehenden gedichte 'The five Joys of the Virgin', Old Engl. Misc. VI, s. $87 \mathrm{f}$. Hier zeigt nämlich a vier hebungen mit klingendem ausgange (unverschliffen) in den strophen I. IV. VI. VII, b hat hier vier hebungen stumpf, zweisilbig in strophe I. Alle zeilen haben vier hebungen stumpf in str. II, vier hebungen stumpf in a (zveisilbig), drei hebungen klingend in $\mathrm{b}$ hat strophe V. In strophe III endlich haben alle zeilen drei hebungen klingend. 
18. Vier hebungen in allen zeilen hat das gedicht 'A luue Ron', Old Engl. Misc. X, s. 93 ff. Hicr hat a vier hebungen klingend, b vier hebungen stumpf in den strophen I. II. III. V. VI. VII. IX. X. XIII. XIV. XV. XVI. XXI. XXII. XXIV. XXV. XXVI; alle zeilen haben vier hebungen klingend strophe XII (betonung: 89 A swetc if pic icnónc, $95 \mathrm{Ne}$ jürhte pé néuer rérve); vier hebungen stumpf str. XI (betonung: ás hénry úre king), zweisilbig str. VIII; a vier hebungen stumpf, b vier hebungen klingend str. XVIII. $\mathrm{XX}$; alle vier hebungen klingend str. XXII.

In diesen beiden zuletzt genannten gedichten ist also mit der neuen form auch das alte versprinzip iiberwunden, das sich in den bisher betrachteten metren noch wirksam erhalten hatte.

WIESBADEN.

ThEODOR WISSMANN. 